\title{
Nonlinear temperature effects on multifractal complexity of metabolic rate of mice
}

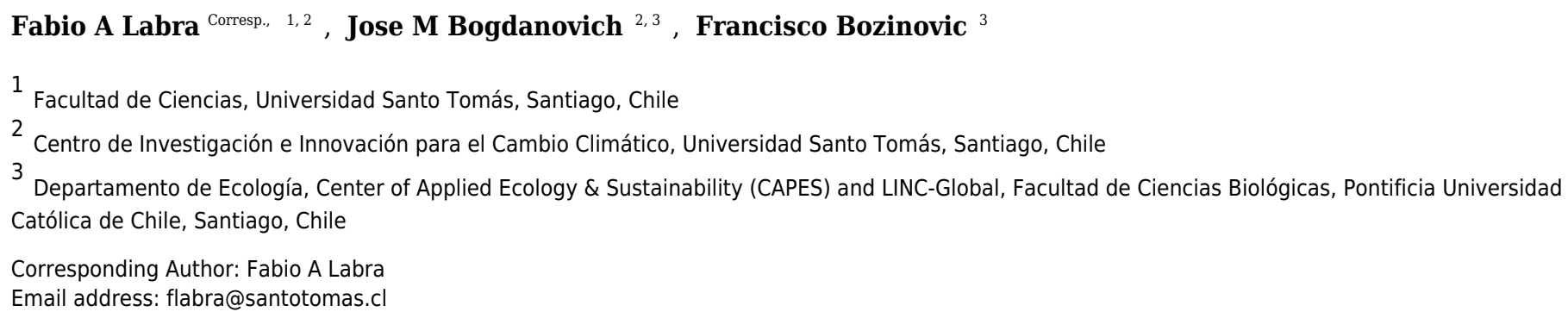

Complex physiological dynamics have been argued to be a signature of healthy physiological function. Here we test whether the complexity of metabolic rate fluctuations in small endotherms decreases with lower environmental temperatures. To do so we examine the multifractal temporal scaling properties of the rate of change in oxygen consumption $r\left(\mathrm{VO}_{2}\right)$, in the laboratory mouse Mus musculus, assessing their long range correlation properties across 7 different environmental temperatures, ranging from $0^{\circ} \mathrm{C}$ to $30^{\circ} \mathrm{C}$. To do so, we applied multifractal detrended fluctuation analysis (MF-DFA), finding that $r\left(\mathrm{VO}_{2}\right)$ fluctuations show two scaling regimes. For small time scales below the crossover time (approximately $10^{2}$ seconds), either monofractal or weak multifractal dynamics are observed depending on whether $T_{a}<15^{\circ} \mathrm{C}$ or $T_{a}>15^{\circ} \mathrm{C}$ respectively. For larger time scales, $r\left(\mathrm{VO}_{2}\right)$ fluctuations are characterized by an asymptotic scaling exponent that indicates multifractal anti-persistent or uncorrelated dynamics. For both scaling regimes, a generalization of the multiplicative cascade model provides very good fits for the Renyi exponents $\tau(q)$, showing that the infinite number of exponents $h(q)$ can be described by only two independent parameters, $a$ and $b$. We also show that the long-range correlation structure of $r\left(\mathrm{VO}_{2}\right)$ time series differs from randomly shuffled series, and may not be explained as an artifact of stochastic sampling of a linear frequency spectrum. These results show that metabolic rate dynamics in a well studied micro-endotherm are consistent with a highly non-linear feedback control system. 
1 Nonlinear temperature effects on multifractal complexity of metabolic rate of mice

2 Fabio A. Labra ${ }^{1,2}$, José M. Bogdanovich ${ }^{2,3, * *}$ and Francisco Bozinovic ${ }^{3 *}$.

3 'Facultad de Ciencias, Universidad Santo Tomas, Ejercito 146, Código Postal 8370003, 4 Santiago, Chile.

5 2Centro de Investigación e Innovación para el Cambio Climático (CIICC), Universidad Santo 6 Tomás, Ejercito 146, Código Postal 8370003, Santiago, Chile..

7 32Departamento de Ecología, Center of Applied Ecology \& Sustainability (CAPES) and LINC-

8 Global, Facultad de Ciencias Biológicas, Pontificia Universidad Católica de Chile, Código Postal 96513677 , Chile.

10 *Corresponding Author;

11 Fabio A. Labra ${ }^{1,2}$

12 Ejército 146, Código Postal 8370003, Santiago, Chile

13 Email address: flabra@santotomas.cl

$14 * *$ Former José M. Rojas 


\section{Abstract}

17 Complex physiological dynamics have been argued to be a signature of healthy physiological

18 function. Here we test whether the complexity of metabolic rate fluctuations in small endotherms

19 decreases with lower environmental temperatures. To do so we examine the multifractal

20 temporal scaling properties of the rate of change in oxygen consumption $r\left(\mathrm{VO}_{2}\right)$, in the

21 laboratory mouse Mus musculus, assessing their long range correlation properties across 7

22 different environmental temperatures, ranging from $0^{\circ} \mathrm{C}$ to $30^{\circ} \mathrm{C}$. To do so, we applied

23 multifractal detrended fluctuation analysis (MF-DFA), finding that $r\left(\mathrm{VO}_{2}\right)$ fluctuations show two

24 scaling regimes. For small time scales below the crossover time (approximately $10^{2}$ seconds),

25 either monofractal or weak multifractal dynamics are observed depending on whether $T_{a}<15^{\circ} \mathrm{C}$

26 or $T_{a} \geq 15^{\circ} \mathrm{C}$ respectively. For larger time scales, $r\left(V O_{2}\right)$ fluctuations are characterized by an

27 asymptotic scaling exponent that indicates multifractal anti-persistent or uncorrelated dynamics.

28 For both scaling regimes, a generalization of the multiplicative cascade model provides very

29 good fits for the Renyi exponents $\tau(q)$, showing that the infinite number of exponents $h(q)$ can be

30 described by only two independent parameters, $a$ and $b$. We also show that the long-range

31 correlation structure of $r\left(\mathrm{VO}_{2}\right)$ time series differs from randomly shuffled series, and may not be

32 explained as an artifact of stochastic sampling of a linear frequency spectrum. These results

33 show that metabolic rate dynamics in a well studied micro-endotherm are consistent with a

34 highly non-linear feedback control system. 
37 Physiologic complexity is ubiquitous in all living organisms (West et al. 1994; Glass 2001;

38 Golberger et al. 2002; Burggren \& Monticino 2005). It emerges as the result of interactions

39 among multiple structural units and regulatory feedback loops, all of which function over a wide

40 range of temporal and spatial scales, allowing the organism to respond to the stresses and

41 challenges of everyday life (West et al. 1994; Goldberger et al. 2002). As a consequence of these

42 intricate regulation feedbacks, most physiological state variables typically present non-linear,

43 non-stationary dynamics, with irregular fluctuations that follow power-law probability

44 distributions and present long-range correlations over multiple time scales (Glass 2001;

45 Goldberger \& West 1987; Kantelhardt 2011; Labra et al. 2007; Mantegna \& Stanley 2000; West

46 et al. 1994). The application of analytic techniques from nonlinear dynamics and statistical

47 physics to the study of different physiologic variables has led to the proposition of a general

48 theory to account for the complexity of physiologic variables (Glass 2001; Costa et al. 2002;

49 Goldberger et al. 2002; Kantelhardt 2011; Lipsitz 2004). This theory states that, given certain

50 parameter conditions, the state variables of healthy systems reveal complex variability associated

51 with long-range (fractal) correlations, along with distinct classes of nonlinear interactions

52 (Goldberger 1996; Goldberger et al. 1990; Goldberger et al. 2002). Over the last two decades,

53 different studies have shown that the break down of this type of multi-scale, nonlinear

54 complexity is a characteristic signature of disease and senescence, and as a result, the study of

55 complexity in physiological variables has shown important promise in the efforts to understand

56 and diagnose different pathologies (Costa et al. 2008; Delignières \& Torre 2009; Goldberger et

57 al. 2002; Hausdorff et al. 2001; Hu et al. 2004; Ivanov et al. 2007; Lipsitz 2004). 
58 While different quantitative approaches have been devised to measure the degree of complexity

59 in physiological signals (e.g. Burggren \& Monticino 2005; Costa et al. 2002; Feldman \&

60 Crutchfield 1998; Pincus 1991; Rezek \& Roberts 1998; Richman \& Moorman 2000; Schaefer et

61 al. 2014), most studies examining changes in physiological complexity as a result of pathological

62 alterations have been conducted by examining either the change or loss of long-range

63 correlations of physiologic signals (e.g. Costa et al. 2008; Delignières \& Torre 2009; Goldberger

64 et al. 2002; Hausdorff et al. 2001; Hu et al. 2004; Ivanov et al. 2007; Lipsitz 2004). Long-range

65 correlated time series typically exhibit slowly decaying auto-correlation functions $C(s)$ across

66 different time scales $s$, which are characterized by power law decay:

with scaling exponent taking values in the range $0<\gamma<1$, such that a characteristic correlation time scale cannot be defined (Chaui-Berlinck et al., 2002a; Chaui-Berlinck et al., 2002b; Billat et al., 2006; Kantelhardt 2011). It has been argued that the lack of a characteristic scale in physiological systems may help the organism to be more stable and adaptive to internal and external perturbations by preventing the emergence of periodic behaviors or phase locking, thus avoiding any restriction to the functional responsiveness of the organism in the face of external perturbations (Peng et al. 1993; Peng et al. 2002, West \& Shlesinger 1989). If this were correct, the study of long-range correlations would provide important insights on the degree of regulation and homeostasis of living organisms, as well as potential tools in the diagnosis of certain pathologies. A power law scaling of the spectrum of Fourier frequencies may also describe the presence of long-term correlations in any given stationary physiological signal: 
80 Long-range correlated processes of this type are often referred to as $1 / f^{\beta}$ processes or noises, and

81 are characterized by a unique value of the scaling exponent $\beta$, which provides a measure of the 82 type of long-range correlation (Chaui-Berlinck et al., 2002a; Chaui-Berlinck et al., 2002b; Billat 83 et al., 2006; Kantelhardt 2011; Schaefer et al 2014). Again, the power law scaling implies that no 84 single characteristic scale may be identified. The Fourier power spectrum scaling exponent may 85 be related to the correlation function exponent by the relationship $\beta=1-\gamma$. Further, the different scaling exponent values are associated with different types of correlation structure in a given

87 time series or signal. Thus, for processes where $\beta=0$ (or $\gamma=-1$ ) the signal shows no long-range correlation between values, while values where $\beta>0$ (or $\gamma>-1$ ) describe a process with longrange correlation or persistence. Processes where $\beta<0$ (or $\gamma<-1$ ) describe a signal with longrange anti-correlations, or anti-persistence, where large values are followed by small ones (Witt \& Malamud 2013). Nevertheless, the use of frequency spectra requires not only that the time series be stationary, but also the use of particular binning procedures as well as averaging over a large number of realizations in order to accurately estimate the value of the scaling exponent $\beta$ (Kantelhardt 2011; Witt \& Malamud 2013). An alternative approach for non-stationary time series is to characterize its long-range persistence by examining the self-affinity of the profile or cumulative sum $z_{i}=\sum r\left(V O_{2}, i\right)$, for all samples $i=1$ to $N$ (Peng et al. 2002, Kantelhardt 2011).

97 Examination of these time series requires us to take into account that the time axis and the axis of the measured values $x(t)$ are not equivalent quantities, and that a rescaling of time $t$ by a factor $a$ may require rescaling of the series values $x(t)$ by a different factor $a^{H}$ in order to obtain a signal 
101 self-affinity or statistical self-similarity in a time series may be described by the resulting scaling

102 relation $x(t) \rightarrow a^{H} x(a t)$ where $H$ corresponds to the Hurst exponent, which measures the degree of

103 persistence or predictability of the profile or cumulated time series (Kantelhardt 2011). The

104 exponent $H$ may be studied by different methods including rescaled range analysis, fluctuation

105 analysis, and detrended fluctuation analysis (Peng et al. 2002, Kantelhardt 2011). In particular,

106 Detrended fluctuation analysis (DFA) has been widely employed to reliably detect long-range

107 autocorrelations in non-stationary time series, with a large number of studies using it to report

108 long-range autocorrelations, although a few studies have reported anti-persistent anti correlations

109 (e.g. Bahar et al. 2001; Delignières et al. 2006, 2011; Kantelhardt 2011). The value of the Hurst

110 exponent $H$ may be approximated by the DFA, which calculates the scaling of mean-square

111 fluctuations with time series scale, yielding the scaling exponent $\alpha$ (Feder 1988; Hurst 1951;

112 Peng et al. 2002, Kantelhardt 2011). When DFA scaling relationships are observed, the scaling

113 exponent $\alpha \approx \mathrm{H}$ is related to the correlation exponent $\gamma$ by the relationship $\alpha=1-\gamma / 2$, with $\alpha=0.5$

114 being the threshold between anti persistence and persistence (Peng et al. 2002, Kantelhardt 115 2011).

117 Despite the increased interest to study fractal or long-range correlated dynamics across many

118 systems, in some highly nonlinear complex systems, the resulting time series presents a scaling

119 autocorrelation function and frequency power spectrum which may be better described by a large

120 number of scaling exponents rather than by a single scaling exponent value (Kantelhardt 2011).

121 Thus, one may distinguish between monofractal and multifractal signals. Monofractal signals

122 present a long-range correlation structure where a single scaling exponent suffices to describe the 
123 correlation scaling. On the other hand, multifractal signals require an infinite spectrum of scaling

124 exponents to describe their correlation structure (Humeau et al. 2009; Ivanov et al. 1999;

125 Kantelhardt 2011; Suki et al. 2003; West \& Scafetta 2003). Thus, multifractal time series are

126 heterogeneous, showing a given value of the self-affinity exponent only in local ranges of the

127 signal structure, such that their self-affinity exponent varies in time. Hence, multifractal signals

128 may be characterized by a set of local fractal sets that represent the support for each Hurst

129 exponent value (Bassingthwaighte et al. 1994; Ivanov et al. 1999; Kantelhardt 2011). In this

130 regard, multifractal time series are more complex than monofractal ones, and determining

131 whether a given complex physiologic system presents monofractal or multifractal dynamics may

132 provide insight on the degree of complexity or nonlinearity of the underlying control

133 mechanisms (Mantegna \& Stanley 1997).

134 In endotherms, metabolic rate $\left(\mathrm{VO}_{2}\right)$ is a global emergent property that reflects the sum of the

135 energetic costs required to maintain homeostasis, allowing body temperature $(\mathrm{Tb})$ to remain as

136 constant as possible despite any changes of its surrounding ambient temperatures ( $\mathrm{Ta}$ ) (Karasov

137 \& Rio 2007; Lighton 2008; McNab 2002). Under controlled laboratory conditions, it is possible

138 to identify a range of optimal $T a$ values where $T b$ may be kept constant without changes in

139 energy expenditure, but rather as a result of adjustments to physical processes (i.e. conductance,

140 radiation, and convection). Within this range of $T a$ values $\mathrm{VO}_{2}$ is expected to show minimal

141 variation, and hence it is named the thermo-neutral zone (TNZ) (Bozinovic \& Rosenmann 1988;

142 Chaui-Berlinck et al. 2005; Karasov \& Rio 2007; Lighton 2008; Lipsitz 2004; McNab 2002). A

143 striking characteristic of $\mathrm{VO}_{2}$ signals is that, even within the $T N Z$, they may be non-stationary,

144 showing changes in the mean and variance of the time series (Chaui-Berlinck et al. 2002a).

145 Studies with small endotherms have shown that $V \mathrm{O}_{2}$ dynamics within the $T N Z$ present irregular 
146 fluctuations with long-range correlations, evidenced by the presence of a single monofractal $1 / f^{\beta}$

147 scaling exponent in the Fourier frequency spectrum (Chaui-Berlinck et al., 2002a; Chaui-

148 Berlinck et al., 2002b; Billat et al., 2006). Thus, within the $T N Z, V \mathrm{O}_{2}$ shows complex dynamics

149 that are consistent with a dynamical system under non-linear control (Chaui-Berlinck et al.

150 2005). The non-stationary behaviour in metabolic rate may be examined by analysing the rate of

151 change in oxygen consumption, $r\left(\mathrm{VO}_{2}\right)$ as a measure of the fluctuations of $\mathrm{VO}_{2}$. It is defined as

$152 r\left(\mathrm{VO}_{2}\right)=\log 10\left[\mathrm{VO}_{2}(\mathrm{t}+1) / \mathrm{VO}_{2}(t)\right]$ (Labra et al. 2007). This variable reveals whether clusters of

153 large, abrupt changes may be seen in the $r\left(\mathrm{VO}_{2}\right)$ time series, or if similar variability is observed

154 throughout. In addition, the calculation of $r\left(\mathrm{VO}_{2}\right)$ allows the de-trending of the data, yielding a

155 much more stationary time series. Examination of $r\left(\mathrm{VO}_{2}\right)$ time series for different species of

156 small mammals, birds and reptiles have shown that this variable has a symmetric power law

157 probability distribution, centered in $r\left(\mathrm{VO}_{2}\right)=0$, with a universal triangular shape that does not

158 change across different species (Labra et al. 2007). Thus, metabolic rate fluctuations follow a

159 single statistical distribution despite differences in cardiovascular and respiratory designs, with

160 distribution width scaling inversely with individual body size (Labra et al. 2007). However, to

161 date, the correlation structure in $r\left(\mathrm{VO}_{2}\right)$ has not been examined. In a similar fashion to other

162 complex non-linear time series, long-term correlations in $r\left(\mathrm{VO}_{2}\right)$ would mean that large

163 fluctuations are more likely to be followed by another large oscillation, while a small oscillation

164 is likely to be followed by a small oscillation (Ashkenazy et al. 2003; Bunde \& Lennartz 2012).

165 If this were the case, the expected average value of $\mathrm{VO}_{2}$ would increase, showing a persistent

166 trend. For $\mathrm{VO}_{2}$ to show homeostatic regulation however, its fluctuations would be expected to

167 show anti-persistence over at least at some scales, so that large $r\left(\mathrm{VO}_{2}\right)$ increases may be followed

168 by large $r\left(\mathrm{VO}_{2}\right)$ decreases, ensuring that overall average $\mathrm{VO}_{2}$ values remain under homeostatic 
169 control. Thus, the presence of anti-persistent correlations may be expected for $r\left(\mathrm{VO}_{2}\right)$ time series,

170 particularly if there are strong control feedback loops regulating total energy expenditure in an

171 organism. This suggests that examination of the type of autocorrelations present in $r\left(\mathrm{VO}_{2}\right)$ time

172 series, as well as the range of time scales involved may provide insight on the regulation

173 feedback that may be acting on metabolic rate at the level of the organism. To gain some

174 understanding of how this may be so, we examine the relationship between thermal stress and $175 \mathrm{VO}_{2}$ fluctuations.

177 In endotherms, $\mathrm{VO}_{2}$ fluctuations are expected to be proportional to the environmental thermal 178 challenges, measured as changes in the difference $(T b-T a)$ (Bozinovic \& Rosenmann 1988;

179 Chaui-Berlinck et al. 2005; Karasov \& Rio 2007; Lighton 2008). Outside the TNZ, adjustments

180 to the body's thermal conductance are not enough to sustain thermal homeostasis, and 181 consequently additional physiological and biochemical process are required in order to keep 182 constant the internal state, which leads to an increase both $\mathrm{VO}_{2}$ and presumably $r\left(\mathrm{VO}_{2}\right)$ as well.

183 In the case of small endotherms, their body size leads to higher challenges associated to the loss 184 of temperature resulting from the large body surface through radiation (Chaui-Berlinck et al. 185 2005; Karasov \& Rio 2007; Lighton 2008; Lipsitz 2004; McNab 2002). Given the intricate 186 nature of the network of control processes involved in achieving constant $T b$ (Chaui-Berlinck et 187 al. 2005), it is reasonable to expect that when faced with lower environmental temperatures 188 values below the $T N Z$, endothermic homeostatic processes would be accompanied by a more 189 complex pattern of auto-correlations. To determine whether this is the case, we use fractal and 190 multifractal analysis to examine whether the correlation structure of $\mathrm{VO}_{2}$ shows any changes as a 
191 result of decreasing environmental temperatures. In this regard, a working hypothesis is that for

$192 \mathrm{Ta}$ values below the $\mathrm{TNZ}$ the $r\left(\mathrm{VO}_{2}\right)$ signal should show a more complex pattern of long-range

193 correlations, resulting in a broader range of autocorrelation scaling exponents, as expected for

194 multifractal signals. These changes should come about as a result of the activation of internal

195 feedback mechanisms to regulate $T b$. A related question to this prediction concerns the form of

196 this possible relation between complexity and decreasing of $\mathrm{Ta}$. Records in wild rodents show a

197 monotonic and linear increment of average $\mathrm{VO}_{2}$ in animals exposed to $\mathrm{Ta}$ decreasing $\left(30^{\circ} \mathrm{C}\right.$ to

$1980^{\circ} \mathrm{C}$ ) (Bozinovic \& Rosenmann 1988), suggesting that $\mathrm{VO}_{2}$ and $r\left(\mathrm{VO}_{2}\right)$ complexity levels may

199 also increase linearly. An alternative outcome may be the gradual decrease and eventual loss of

200 complexity, due to a drop in the efficiency of the thermoregulatory feedback control at lower

201 temperatures (Angilletta 2006; McNab 2002). This second pattern would be in agreement with

202 the hypothesis of loss of physiological complexity in the face of extreme system degradation or

203 acute stress (Goldberger et al. 2002). To test these hypotheses we examine the fractal properties

204 of time series of $r\left(V_{2}\right)$ measurements $\mathrm{n}$ laboratory mice (Mus musculus) exposed to

205 environmental temperatures ranging from $\operatorname{TNZ}\left(30^{\circ} \mathrm{C}\right.$ in this species $)$ to $0^{\circ} \mathrm{C}$. Thus, as first step in

206 this work we assess whether $r\left(V_{2}\right)$ values exhibit either monofractal or multifractal long-term

207 correlations under different environmental temperatures. We do this by testing whether

208 metabolic rate fluctuations show any long-range correlations, and if so, testing whether there

209 may be described either by a single scaling exponent or if multiple scaling exponents are

210 required, using the multifractal detrended fluctuation analysis (MF-DFA) method. We then

211 assess how these quantitative descriptors of long-range correlations vary with environmental

212 temperature, assessing how they change with decreasing values of $\mathrm{Ta}$. 


\section{Methods}

\section{Determination of Metabolic rate}

216 Empirical $\mathrm{VO}_{2}$ time series were determined by measuring metabolic rate in wild-type male white

217 laboratory mice. Mice were transferred to the laboratory and housed individually with sawdust

218 bedding. Mice were provided with water and fed with food pellets ad libitum. Ambient

219 temperature and photoperiod were held constant at $20 \pm 2^{\circ} \mathrm{C}$ and $12 \mathrm{~L}: 12 \mathrm{D}$ respectively. Care of

220 experimental animals was in accordance with institutional guidelines. The Bioethics

221 commissions of Universidad Santo Tomás, Pontificia Universidad Católica de Chile, and The

222 Chilean National Committee of Science and Technology (CONICYT) approved all experimental

223 protocols followed. Animals were held under these conditions for two weeks prior to

224 measurements and then fasted for $3 \mathrm{~h}$ immediately prior to metabolic rate records in metabolic

225 chambers (Lighton 2008). Individuals were measured at seven different $\mathrm{Ta}, 0^{\circ} \mathrm{C}, 5^{\circ} \mathrm{C}, 10^{\circ} \mathrm{C}$,

$22615^{\circ} \mathrm{C}, 20^{\circ} \mathrm{C}, 25^{\circ} \mathrm{C}$ and $30^{\circ} \mathrm{C}$, with the latter corresponding to the lower limit of $T N Z$ in this

227 species. Overall, 18 individuals were assigned to different temperature treatments, with the order

228 of temperature treatments for each individual assigned at random to avoid any artefacts. In

229 addition, colonic body temperature $\left(\mathrm{T}_{\mathrm{b}}\right)$ was recorded at the end of each measurement using a

230 Digi-Sense copper-constant thermocouple to evaluate a possible torpor condition at the end of

231 the experiment. In each experimental record $\mathrm{VO}_{2}$ was measured in a computerized open-flow

232 respirometry system (Sable Systems, Las Vegas, Nevada). The metabolic chamber received dried

233 air at a rate of $800 \mathrm{ml} / \mathrm{min}$ from mass flow-controllers (Sierra Instruments ${ }^{\mathrm{TM}}$, Monterey,

234 California), which ensured adequate mixing in the chamber. Air passed through $\mathrm{CO}_{2}$ and $\mathrm{H}_{2} \mathrm{O}$

235 absorbent granules of Baralyme ${ }^{\mathrm{TM}}$ and Drierite ${ }^{\mathrm{TM}}$ respectively before and after passing through

236 the chamber and was monitored every $1 \mathrm{sec}$. This allowed us to obtain time series of oxygen 
237 consumption recorded at periodic intervals of $t=1$ second. After the $r\left(\mathrm{VO}_{2}\right)$ time series were

238 registered, they were then analysed by calculating the corresponding $r\left(\mathrm{VO}_{2}\right)$ time series.

239 Assessing long range correlations in metabolic rate

240 To determine the presence of long-term correlations in the $r\left(V_{2}\right)$ time series, we examined the

241 power spectral density $\left.S(f) \equiv x(f)\right|^{2}$, where $x(f)$ is the Fourier transform of $r\left(V O_{2}\right)$ data

242 observations measured under experimental conditions $\left(x_{i}\right)$ evaluated at frequencies $f=0, \ldots, N / 2$

243 (Bunde \& Lennartz 2012; Kantelhardt 2011). As mentioned above, for long-term correlated time

244 series, it can be shown that the power spectral density decays with frequency following a power

245 law (see Equation 2). In order to avoid potential artefacts due to lack of stationary behaviour, we

246 also used the Detrended Fluctuation Analysis method (DFA) (Kantelhardt 2011; Peng et al.

247 1995a). Briefly, DFA analyses a profile or accumulated data series $z_{i}=\sum r\left(V O_{2}, i\right)$, for all samples

$248 i=1$ to $N$. The profile is divided into $N_{s}$ non-overlapping segments of scale s. For every segment

$249 v$, the local trend is fit by a polynomial of order $n$, and the variance raised to the 2-th power $250\left[\sigma^{2}(v, s)\right]^{2}$ between the local trend and the profile in each segment $v$ is calculated. The mean 251 fluctuation function $F(s)^{2}$ is then calculated by:

252

$$
F_{2}(s)=\left\{\frac{1}{N_{s}} \sum^{\prime}\left[s^{2}(s)\right]\right\}^{1 / 2}
$$

254 (equation 3: $F_{2}(s)=\left[1 / N_{s}\right]\left[\Sigma\left(s^{2}(s)\right)\right]^{1 / 2}$ )

255 Examination of how $F_{2}(s)$ scales with box size or scale $s$ allows the estimation of the scaling 
256 exponent $\alpha_{D F A}$, which is often referred to as the global Hurst exponent $H$ (Goldberger et al. 2002;

257 Ivanov et al. 2007; Kantelhardt 2011; Peng et al. 1995a). When observed time series are either

258 uncorrelated or show short term correlations, $\alpha_{D F A}=0.5$ (Kantelhardt 2011; Peng et al. 1995a).

259 For long-term correlated data with persistent $1 / f^{\beta}$ noise, where $\beta=1.0, \alpha_{D F A}$ exhibits values of

260 equal to 1.0. For values of $\alpha_{D F A}$ below 0.5 , the series is said to be anti-persistent, with positive

261 trends being associated with negative trends (Delignières et al. 2006, 2011).

262 Assessing multifractality of metabolic rate

263 To determine the presence of multifractality in the fluctuations of metabolic rate we applied 264 multifractal detrended fluctuation analysis (MF-DFA) (Kantelhardt 2011; Kantelhardt et al. 265 2002) to $r\left(\mathrm{VO}_{2}\right)$ data measured under experimental conditions. This method yields similar 266 results to other existing methods of multifractal analysis in time series (Ivanov et al. 2007; 267 Kantelhardt 2011; Kantelhardt et al. 2002; Ludescher et al. 2011; Oswiecimka et al. 2006), but is 268 considerably easier to implement, being based on an extension of DFA (Kantelhardt 2011; 269 Kantelhardt et al. 2002; Ludescher et al. 2011). Briefly, MF-DFA analyses a profile or 270 accumulated data series $z_{i}=\sum r\left(V O_{2}, i\right)$, for all samples $i=1$ to $N$. The profile is divided into $N_{s}$ 271 non-overlapping segments of scale $s$. For every segment $v$, the local trend is fit by a polynomial 272 of a given order $o$, where $o=1,2$ or 3 . The resulting variance is then raised to the $\mathrm{q} / 2$-th power $273\left[\sigma^{2}(v, s)\right]^{q / 2}$ between the local trend and the profile in each segment $v$ is calculated. When $q=0$, 274 logarithmic averaging may be applied (Kantelhardt 2011; Kantelhardt et al. 2002; Ludescher et 275 al. 2011). A generalized fluctuation function $F_{q}(s)$ is then calculated by averaging all the 276 variances across all segments of scale $s$ : 
278

279

280

281

282

283

284

285

286

287

288

289

290

291

292

293

294

295

296

( equation 4: $\left.F_{q}(s)=\left[1 / N_{s}\right]\left[\Sigma\left(\sigma^{2}(v, s)\right)\right]^{q / 2}\right)^{1 / q}$

In general, $F_{q}(s)$ exhibits a scaling relationship with time scale $s: F_{q}(s) \sim s^{h(q)}$, which allows the estimation of a set of exponents $h(q)$ for every moment q. These scaling exponents correspond to the generalized Hurst exponents. In some nonlinear complex systems, the $F_{q}(s)$ function has been shown to exhibit scaling crossovers, with more than one asymptotic scaling exponent (Koscielny-Bunde et al. 2006). Hence, we tested whether linear or piecewise linear regressions best fit the scaling relationship of $F_{q}(s)$ with $s$, using log-transformed data. The piecewise or segmented relationship between the mean response $\mu=\mathrm{E}[\mathrm{Y}]$ and the variable $\mathrm{X}$, for observation $\mathrm{i}=1,2, \ldots, \mathrm{n}$ was modeled by adding the following terms in the linear predictor:

$\beta_{0}+\beta_{1} X_{i}+\beta_{2}\left(X_{i}-\delta\right)+$

where $\left(\mathrm{X}_{\mathrm{i}}-\delta\right)+=\left(\mathrm{X}_{\mathrm{i}}-\delta\right) \times \mathrm{I}\left(\mathrm{X}_{\mathrm{i}}>\psi\right)$, and $\delta$ is the fitted breakpoint or crossover point and $\mathrm{I}(\cdot)$ is an indicator function that is equal to one when the statement is true and is equal to zero when the statement is false (Muggeo 2003). Piecewise linear models were fitted using the segmented library (Muggeo 2003) in the R program (R Development Core Team 2014, available at www.rproject.org). If no crossovers were observed, then linear regression would be favored over a piecewise regression. To test this, the segmented library uses Davie's test to test for a nonconstant regression parameter in the linear predictor (Muggeo 2003). Once the correct regression model is identified, the regression slopes provide the asymptotic estimates for the scaling exponents $h(q)$. If no crossover is present, only one scaling exponent $h(q)$ is obtained for every 
297 moment $q$. If a crossover point is detected, then two scaling exponents $h(q)$ and $h(q)$ are obtained 298 for every moment $q$.

299 For monofractal self-affine time series, $h(q)$ is independent of the chosen moment $q$, and 300 is identical to the global Hurst exponent $H$ regardless of the value of the moment $q$ (Feder 1988;

301 Hurst 1951; Kantelhardt et al. 2003; Kantelhardt et al. 2002). Hence, for monofractal self-affine

302 time series $\alpha_{D F A} \approx H$. On the other hand, in multifractal time series $h(q)$ varies with $q$, reflecting

303 the fact that small and large fluctuations scale differently (Kantelhardt et al. 2002). For negative

304 values of $q, h(q)$ describes the scaling behaviour of those time series segments with small

305 fluctuations, whereas for positive values of $q, h(q)$ describes the scaling behaviour of those time

306 series segments with large fluctuations (Kantelhardt et al. 2002). It has been shown that the

307 generalized Hurst exponent $h(q)$ can be directly related to the classical multifractal scaling Renyi

308 exponents $\tau(q)$ defined by the standard partition function-based formalism using the

309 relationships: $\tau(q)=q h(q)-1$ and $h(q)=(\tau(q)+1) / q$ (Kantelhardt et al. 2002; Koscielny-

310 Bunde et al. 2006). Thus, it may be shown for normalized, stationary time series that the

311 multifractal spectra estimated by MF-DFA have a deep similarity with thermodynamics

312 (Kantelhardt et al. 2002).

313 For monofractal records, $\tau(q)$ is a linear function of $q$, while multifractal records are

314 characterized by non-linear dependence of $\tau(q)$ on $q$ (Ivanov et al. 1999; Kantelhardt et al. 2002;

315 Koscielny-Bunde et al. 2006). Also, it can be shown that $h(q)$ may be related to the singularity

316 spectrum $f(\alpha)$ via a Legendre transform:

317

$$
f(\alpha)=q[\alpha-h(q)]+1
$$


318 where $\alpha=[d \tau(q) / d q]$ is the singularity strength, or Hölder exponent, while $f(\alpha)$ denotes the

319 singularity dimension of the subset of the time series that is characterized by a given value of

320 singularity strength $\alpha$ (Feder 1988; Kantelhardt et al. 2002; Ludescher et al. 2011, Ihlen 2012).

321 For monofractal self affine signals, the singularity spectrum of the time series is a single point,

322 showing that there is a unique value or a very small set of values of singularity strength $\alpha$, with a

323 corresponding fractal dimension $f(\alpha)=1$. For multifractal self affine signals, the singularity

324 spectrum of the time series is a parabola, with a maximum at the dominant singularity strength

325 observed in the time series.

326 To assess multifractality in $r\left(\mathrm{VO}_{2}\right)$ time series, we calculated the fluctuation function $F_{q}(s)$ for

327 data obtained from wild-type white laboratory mice $r\left(\mathrm{VO}_{2}\right)$ time series measured under 328 controlled conditions. Following recent studies, we fit both the $h(q)$ and $\tau(q)$ spectra with a 329 modified version of the multiplicative cascade model, which has been proposed by (Koscielny330 Bunde et al. 2006):

$$
h(q)=(1 / q)-\left(\ln \left(a^{q+} b^{q}\right)\right) /(q \ln (2))
$$

332 and

$$
\tau(q)=-\left(\ln \left(a^{q+}+b^{q}\right)\right) /(\ln (2))
$$

334 The modified multiplicative cascade model functions (MMCM) allows the description of

335 multifractal spectra with only two parameters, $a$ and $b$, which take values between 0 and 1 with $a$ $336+b \geq 1$. An additional advantage is that these functions also extend to negative $q$ values, and 337 thus allow estimation of the multifractal spectrum $f(\alpha)$ for these values as well (Koscielny-Bunde 338 et al. 2006). Using the $\tau(q)$ spectra, we estimated the parameters $a$ and $b$ for eqn. (7), allowing us 
339 to obtain continuous $\tau(q)$ and $f(\alpha)$ spectra from the MMCM fits.

340 To test whether observed long term correlation behaviour was different from a random

341 expectation, we randomized all time series using an amplitude-adjusted Fourier transform

342 algorithm (AAFT) (Schreiber \& Schmitz 1996; Schreiber \& Schmitz 2000). The scaling

343 functions were calculated for all surrogate time series and the corresponding scaling exponents

344 (e.g. $\beta$ and $\alpha_{D F A}$ for Fourier spectral density and DFA respectively) were calculated (Schreiber \&

345 Schmitz 1996; Schreiber \& Schmitz 2000).

346 Assessing the effect of temperature on multifractality of metabolic rate fluctuations

347 As explained above, regular $\mathrm{VO}_{2}$ time series were obtained under temperature-controlled

348 conditions (see Methods sections for details). To assess the effect of $T a$ on long range and

349 multifractal measures of $r\left(\mathrm{VO}_{2}\right)$ fluctuations, we calculated the average fluctuation function $F_{q}(s)$

350 for each of the seven temperature treatment groups, testing whether the resulting $h(q)$ and $\tau(q)$

351 spectra are also multifractal. In order to summarize the observed results, we calculated the

352 singularity spectrum $f(\alpha)$, which allows a compact description of the degree of multifractality

353 through the quantification of $\Delta \alpha$, the width of the singularity spectrum as well as the average

354 dominant exponent $\alpha_{\max }$, which indicates which is the dominant scaling exponent, or the one

355 which shows greater support on average across the time series. We then summarized the various

356 spectra across the experimental temperature treatments, allowing us to examine their response to

357 temperature. To test whether observed multifractal behaviour was different from a random

358 expectation, we randomized all time series using an amplitude-adjusted Fourier transform

359 algorithm (AAFT) (Schreiber \& Schmitz 1996; Schreiber \& Schmitz 2000). After the surrogates

360 were generated, the general fluctuation function $F_{q}(s)$ and the $h(q)$ spectra were calculated as 
361 explained above. We then compared $h(q), \tau(q)$ and $f(\alpha)$ spectra for the shuffled time series.

362 Again, we summarized the various spectra for shuffled time series across the experimental

363 temperature treatments, allowing us to compare them with original time series spectra as for

364 different temperature treatments. To assess the potential effect of de-trending polynomial order

$365 o$, all data analyses were carried out for each individual time series were carried out using three

366 orders: $o=1,2$ or 3. Data analyses were carried out using Matlab R2011b and R software (R

367 Development Core Team 2014, available at www.r-project.org).

\section{Results}

371 As described in the physiological literature for endotherms, average $\mathrm{VO}_{2}$ values in the lab mouse

372 show a marked thermal response below $T N Z$, with higher $\mathrm{VO}_{2}$ values that increase away from

373 basal metabolic rate $(B M R)$ as $T a$ becomes progressively lower (Figure 1a). None of the animals

374 studied showed signs of torpor either during or after the $\mathrm{VO}_{2}$ measurements, and observed $\mathrm{Tb}$

375 varied from 36.0 to $37.3{ }^{\circ} \mathrm{C}$ across all records. However, even within the $T N Z\left(30^{\circ} \mathrm{C}\right)$, typical

$376 \mathrm{VO}_{2}$ time series exhibit irregular non-stationary fluctuations (Figure $1 \mathrm{~b}$ ). The rate of change

$377 \mathrm{r}\left(\mathrm{VO}_{2}\right)$ yields a de-trended time series, which reveals abrupt changes in $\mathrm{VO}_{2}$, with clusters of

378 large fluctuations separated from clusters of smaller fluctuations(Figure 1c). This suggests the

379 presence of long-term correlation or persistence in these time series. The clustering of large

380 fluctuations is lost when data are shuffled randomly using AAFT (Figure 1d), providing

381 indication that the observed pattern of $r\left(\mathrm{VO}_{2}\right)$ fluctuations may be associated with the

382 autocorrelation structure of the time series (Schreiber \& Schmitz 1996; Schreiber \& Schmitz 
383 2000; Kantelhardt 2011) rather than with the fat tailed probability distribution shown by this

384 variable (Labra et al. 2007). The statistical pattern of autocorrelation in the sequence of large and

385 small fluctuations may be examined by calculating the Fourier frequency power spectra, which

386 reveals the presence of long-term correlations, shown by a 1/f-like scaling exponent (Figure 1e).

387 On the other hand, shuffled time series exhibit a shallower power spectrum, indicating the loss of

388 these long-term correlations (Figure 1e) (Kantelhardt et al. 2002; Schreiber \& Schmitz 1996;

389 Schreiber \& Schmitz 2000). However, while $r\left(\mathrm{VO}_{2}\right)$ time series do not exhibit obvious trends in

390 the mean, they do show changes in variability through time, and as a result may not meet the

391 statistical assumptions of spectral frequency estimation (Kantelhardt 2011). Examination of

392 detrended fluctuation analysis reveals a scaling crossover, with two clear scaling regimes shown

393 by the root mean square fluctuation function $F_{2}(s)$ (Figure 1f). This suggests that a single scaling

394 exponent may not be sufficient to characterize the autocorrelation of $r\left(\mathrm{VO}_{2}\right)$ fluctuations

395 (Kantelhardt et al. 2002). In this time series, the scaling exponent for small time scales (s $<100$

396 seconds), $\alpha_{D F A l}$, indicates the presence of persistent, long-range correlated fluctuations

$397 \quad\left(\alpha_{\text {DFAl }}=0.91\right) \quad$ (Figure 1f). However, for larger time scales $(s>100$ seconds $)$ we see that

398 fluctuations over these time scales are anti-persistent, with the second scaling exponent

$399 \alpha_{\mathrm{DFA} 2}=0.39$ (Eke 2000, Delignières et al. 2006, 2011). As mentioned above, in anti-persistent

400 time series dynamics positive trends are usually followed by negative trends, thus showing a 401 phenomenological signature of control or negative feedback over the rate of change of $\mathrm{VO}_{2}$

402 (Delignières et al. 2011). Shuffling the data results in a loss of the observed crossover scaling

403 behaviour, indicating this is property is not a result of randomness in the pattern of fluctuations

404 (Figure 1f). Thus, we find that $r\left(\mathrm{VO}_{2}\right)$ fluctuations within the TNZ show non-trivial long-range

405 correlations, in agreement with previous observations for $\mathrm{VO}_{2}$ in small endotherms (Chaui- 
406 Berlinck et al., 2002a, 2002b). However, a single scaling exponent does not suffice to describe

407 these long-range correlations.

408

409

When we examined the DFA scaling functions for $r\left(\mathrm{VO}_{2}\right)$ fluctuations both within and

410 outside the $T N Z$, we observe a similar crossover pattern across different temperatures, with

411 average $F_{2}(s)$ scaling functions show a crossover pattern which is similar to that observed in

412 Figure 1f. Hence, observed scaling exponent values for small to intermediate time scales) are

413 consistent with persistent long-range autocorrelations (i.e. $0.5<\alpha_{D F A 1}<1.0$ ) (Figures $2 \mathrm{a}$ to $2 \mathrm{~d}$ ). On

414 the other hand, for intermediate to large scales, the scaling exponent values are consistent with

415 anti-persistent long-range correlations $\left(\alpha_{D F A 2}<0.5\right)$ (Figures 2a to 2d). Shuffling the individual

416 time series results in changes to the $F_{2}(s)$ scaling functions, with average $\alpha_{D F A 1}$ values becoming

417 smaller (Figures 2e to 2h). Examination of the scaling exponent values shows that $\alpha_{D F A 2}$ values

418 do not show large changes for shuffled data (Figure 3). This pattern is observed for linear (Figure

419 3) as well as for quadratic and cubic de-trending orders $o$ (see Supplementary Figure 1). The

420 existence of two scaling regimes for the long-range correlations of $r\left(\mathrm{VO}_{2}\right)$ may be interpreted as

421 evidence that two dominant scaling exponents may suffice to account for the correlation

422 structure of the $r\left(\mathrm{VO}_{2}\right)$ time series. An alternative possibility may be that a continuous spectrum

423 of scaling exponents are required in order to account for the observed pattern of long-term

424 correlations in $\mathrm{VO}_{2}$ fluctuations. If the latter were the case, local scaling exponents would show a

425 large number of possible values.

426 To visualize whether a sample $r\left(\mathrm{VO}_{2}\right)$ time series is consistent with a multifractal process,

427 we examined the changes in the value of local DFA scaling exponent $\alpha_{D F A}$ through time in the 
428 time series shown in Figure 1 (which was measured within the TNZ). We calculated the local 429 value of $\alpha_{D F A}$ as for a moving window placed along the time series. We calculated $\alpha_{D F A}$ values 430 using moving windows of 128, 256 and 512 seconds (Figures 4a, 4b and 4c respectively). All 431 these window sizes correspond to the asymptotic exponent expected for the second scaling 432 regime identified before for this time series (Figure 1f). Observed local $\alpha_{D F A}$ exponent values 433 change through time for all window sizes used, forming an irregular pattern (Figure 4). Further, $434 \alpha_{D F A}$ values range broadly between 0.5 and 1.5 , as shown by the blue lines in Figure 4 . Thus, 435 while in some sections show exponent values close to 1.0 , corresponding to persistent power law 436 long-range correlations, other sections may show values closer to either 1.5 (corresponding to 437 persistent Brownian motion) or to 0.5 (corresponding to uncorrelated fluctuations) (Peng et al. 438 1995b). There are also sections where the local $\alpha_{D F A}$ scaling exponent may take values below 439 0.5, corresponding to anti-persistent fluctuations (Eke 2000, Delignières et al. 2006, 2011). 440 Again, random shuffling of the time series destroys the observed pattern of irregular fluctuations 441 of $\alpha_{D F A}$, with all exponent values clustering around 0.5 , as shown by the red lines in Figure 4. 442 Thus, for this time series, we can see that observed $r\left(\mathrm{VO}_{2}\right)$ fluctuations cannot be characterized 443 by a single scaling exponent, and hence may be multifractal.

To determine whether this is the case, we examined whether the MF-DFA formalism can describe $\mathrm{VO}_{2}$ fluctuations across different environmental temperatures. Figure 5 shows the average MF-DFA generalized fluctuation functions $F_{q}(s)$ calculated from time series measured at $44730^{\circ}, 20^{\circ}, 10^{\circ}$ and $0^{\circ} \mathrm{C}$ (Figures $5 \mathrm{a}, 5 \mathrm{~b}, 5 \mathrm{c}$ and $5 \mathrm{~d}$ respectively). Across all temperatures studied, 448 and for all the values of $q$ examined, observed $F_{q}(s)$ functions show a crossover $\delta$ that defines 449 two scaling regions, as shown by the fitted piecewise linear regressions (shown in black lines) 450 (Figure 5). Shuffling the time series leads to some changes in the crossover pattern, although no 
451 striking overall pattern may be discerned by qualitative examination (Figures 5e to 5f). It must be

452 noted that while the remaining three series for $5^{\circ}, 15^{\circ}$ and $25^{\circ} \mathrm{C}$ are not shown, they show similar

453 patterns. In fact, detailed examination of the average generalized fluctuation functions reveals

454 that $F_{q}(s)$ show the presence of crossover time scales $\delta$ for all temperatures studied, regardless of

455 the order $o$ of the de-trending polynomial used (see supplementary Figures 2 to 8 for detailed

456 results for different de-trending polynomial orders and all temperatures from $0^{\circ} \mathrm{C}$ to $30^{\circ} \mathrm{C}$ ). Thus,

457 for all temperatures examined, regardless of the order of de-trending polynomial used, we

458 observed two scaling regimes are present, with the piecewise break point changing as a function

459 of $q$ in some cases (see supplementary Figure 9). While it could be argued that such scaling

460 crossovers may be the result of trends associated with non-stationary dynamics in the data,

461 examination of the Augmented Dickey-Fuller Test (ADF test) for all $r\left(\mathrm{VO}_{2}\right)$ time series rejected

462 the hypothesis of the presence of trends, and we observed that the ADF test yields $p<0.01$ in all

463 time series. Shuffling of the observed $r\left(V O_{2}\right)$ time series does not completely remove the

464 crossover scales $\delta$ or the two observed regimes, but does seem to change the scaling exponent for

465 the first scaling regime (see supplementary Figures 2 to 8). Given the presence of two scaling

466 regimes across all time series studied, we then examined the scaling slopes of the curves for both

467 of these scaling regimes and their change with the exponents $q$. This allowed us to estimate the

468 average Hurst $(h(q))$ and Renyi $(\tau(q))$ spectra for each of these two scaling regimes. We then also

469 fitted the MMCM model to the observed Renyi $(\tau(q))$ spectra, and estimated the singularity

470 spectra $(f(\alpha))$ based on these parameter fits.

471 When we examined average Hurst $(h(q))$ and Renyi $(\tau(q))$ spectra, as well as the

472 corresponding singularity spectra $(f(\alpha))$ estimated from the MMCM fits on $\tau(q)$, we found that

473 the two scaling regimes differ in their multifractal spectra across the seven temperatures studied. 
474 The left hand column of Figure 6 shows the multifractality of $r\left(\mathrm{VO}_{2}\right)$ fluctuations, as indicated by

475 the dependence of $h(q)$ on $q$ for different temperature values. We find that fluctuations of

476 different magnitudes in $r\left(\mathrm{VO}_{2}\right)$ time series show different scaling behaviour, similar to what has

477 been observed other complex systems (Bunde \& Lennartz 2012; Kantelhardt et al. 2006;

478 Kantelhardt et al. 2002). However, the first and second scaling regimes differ in their behaviour,

479 with smaller time scales (in the approximate range $8 \leq \mathrm{s} \leq 100$ ) showing generalized Hurst

480 exponent $h_{1}(q)$ values closer to 1.5 , while larger time scales (in the approximate range

$481100 \leq \mathrm{s} \leq 1024$ ) show generalized Hurst exponents decreasing from $h_{2}(q) \approx 0.9$ to $h_{2}(q) \approx 0.25$ as the

482 exponent order $q$ increases (Figure 6). Hence, fluctuations on the first scaling regime show long-

483 range correlations or persistence, similar to that of Brownian motion, regardless of the magnitude

484 of the fluctuation. On the other hand, for the second scaling regime, small $\mathrm{VO}_{2}$ fluctuations are

485 characterized by larger scaling exponents $h_{2}(q)$, corresponding to power law, long-range

486 correlated persistent dynamics, while larger $\mathrm{VO}_{2}$ fluctuations present smaller $h_{2}(q)$ exponent

487 values, corresponding to anti-persistent dynamics (see left hand column in Figure 6). Thus, over

488 intermediate to large time scales, large positive $r\left(\mathrm{VO}_{2}\right)$ values are balanced by large negative

489 values. On the other hand, for this range of scales, small $r\left(\mathrm{VO}_{2}\right)$ values are persistent, such that

490 small positive increases are followed by similarly valued changes, resulting in gradual positive

491 trends in $\mathrm{VO}_{2}$. A similar pattern occurs for negative rates of change, which leads to gradual

492 negative trends in $\mathrm{VO}_{2}$. Shuffling the $r\left(\mathrm{VO}_{2}\right)$ time series results in markedly lower values of $h(q)$

493 scaling exponents for the first scaling regime, indicating the observed, persistent long-range

494 correlation cannot be accounted for by a random sample of the observed spectral density

495 function. On the other hand, in the second scaling regime, a complex response is observed,

496 where shuffling results in changes only for negative and small positive $q$ values, whereas 
497 observed exponents for large positive $q$ values overlap with the exponents from shuffled time

498 series. In fact, with the exception of $30^{\circ} \mathrm{C}$, very large fluctuations in $r(V O 2)$ do not differ from

499 the random expectation (Figure 6).

500 Observed differences in the range of $h(q)$ exponents for the two scaling regimes can also be

501 observed when examining the Renyi exponent spectra. We observed mostly linear Renyi

502 exponent spectra in the first scaling regime, while the second scaling regime shows nonlinear

503 Renyi exponent spectra as expected for multifractal time series (Kantelhardt 2011) (see central

504 column, Figure 6). This suggests that the first scaling regime should either be monofractal or

505 weakly multifractal, requiring a smaller range of scaling exponents to account for the observed

506 singularities. On the other hand, the second scaling regime is characterized by strong

507 multifractality, with a broader range of scaling exponent values. As observed in previous results,

508 shuffling destroys the observed scaling spectra, with the exception of $\tau(q)$ values observed for

509 positive $q$, which do not differ from the shuffled spectra (Figure 6). In all the time series we

510 examined, the observed Renyi exponent spectra were fit extremely well my the MMCM model

511 shown in equation 6 , with $\mathrm{R}^{2}$ values for the nonlinear fitting procedure being close to 1.0 in all

512 cases (see Supplementary Figure 10). This allowed us to use the fitted $\tau(q)$ values to estimate the

513 singularity spectra $f(\alpha)$ for each individual, which were then averaged across all the different

514 temperature treatments.

515 Examination of the average singularity spectra $f(\alpha)$ for different temperature treatments shows

516 that the first scaling regime of these $r(V O 2)$ time series are monofractal or weakly multifractal,

517 as evidenced by either a single point or a narrower parabola in the $(\alpha, f(\alpha)$ ) plane (see dashed

518 lines in graphs on the right hand column in Figure 6). These qualitative patterns do not change 
519 when quadratic or cubic de-trending polynomials are used (see right hand columns of

520 Supplementary Figures 11 and 12). Indeed, the average degree of multifractality, $\Delta \alpha$ shows that

521 the first scaling regime the strength of multifractality decreases with temperature (see Figure 7).

522 While a similar qualitative pattern is observed for all de-trending polynomial orders, a the

523 decrease with temperature is significant only for the linear de-trending case (linear OLS

524 regression, $\mathrm{F}=8.202$, d.f. $=(1,5), \mathrm{p}=0.035$ ) (Figures $7 \mathrm{a} 7 \mathrm{~b}$ and $7 \mathrm{c}$ ). In sharp contrast, the second 525 scaling regime shows broad singularity spectra, indicating a much larger degree of 526 multifractality, $\Delta \alpha$ (see continuous lines in graphs on the right hand column in Figure 6). For this 527 second scaling regime, no significant linear trends with temperature were observed, with the 528 exception of the cubic de-trended data (linear OLS regression, $F=13.43$, d.f. $=(1,5), p=0.015)$ 529 (Figure 7c). Shuffled data tend to show similar degrees of multifractality across different 530 temperatures and orders of detrending polynomials (Figure $7 \mathrm{~d}$ to $7 \mathrm{f}$ ).

531 On the other hand, when we examine the exponent $\alpha_{\max }$ of the singularity spectra, we see that 532 the first scaling regime is characterized by much stronger singularities, with $\alpha_{\max }$ taking values 533 closer to 1.5 , being slightly larger for $15^{\circ} \mathrm{C}$ and $20^{\circ} \mathrm{C}$ (Figures 6i and 6o). On the other hand, the 534 second scaling regime is characterized by weaker stronger singularities, showing values of $\alpha_{\max }$ 535 below 0.5 (see right hand column of Figure 6 and Figure 8). Examination of the changes in $\alpha_{\max }$ 536 as a function of temperature for the first scaling regime indicates that the value of $\alpha_{\max }$ has 537 significant increases with temperature only for the linear and cubic cases (linear de-trending: $538 \mathrm{~F}=7.52$, d.f. $=(1,5), \mathrm{p}=0.04$; cubic de-trending: $\mathrm{F}=7.52$, d.f. $=(1,5), \mathrm{p}=0.04)$ (Figure 8a to 8c). In 539 the case of quadratic de-trending, temperature values equal or greater than $15^{\circ} \mathrm{C}$ show high 540 values of $\alpha_{\max }$, coherent with the persistent, Brownian motion-like values of $h(q)$ observed 541 before. On the other hand, for the second scaling regime, $\alpha_{\max }$ does not show significant changes 
542 with temperature for any de-trending order (Figure 8a to 8c). Shuffled data tend to show similar

543 degrees of multifractality for different temperatures and orders of de trending polynomials, with

544 shuffled data for the first scaling regime clustering around values close to $\alpha_{\max }=0.9$, and shuffled

545 data for the second scaling regime clustering around values close to $\alpha_{\max }=0.3$ (Figure $8 \mathrm{~d}$ to $8 \mathrm{f}$ ).

546 Thus, both the observed degree of multifractality $\Delta \alpha$, and the dominant multifractal singularity

547 exponent $\alpha_{\max }$ in these two scaling regimes cannot be attributed to random fluctuations.

\section{Discussion}

Physiological systems, and their state variables and signals, have been recognized as complex (Burggren \& Monticino 2005; Glass 2001). To date, most studies examining the causes and functional implications of the loss of complexity in organisms have largely focused on

553 human biomedicine, aiming to understand either pathologies or the senescence process (Costa et

In this regard, our study aims to provide a better understanding of the role of physiological complexity in the homeostatic response to thermal challenges, particularly in the context of a changing world climate. Here, we analyzed the dynamics of metabolic rate fluctuations, $r\left(\mathrm{VO}_{2}\right)$, under different $\mathrm{Ta}^{\prime}$ s using a well-studied model organism, the lab mouse Mus musculus. Using MF-DFA, our results show that within the $T N Z, r\left(\mathrm{VO}_{2}\right)$ time series show two distinct

560 scaling regimes in the fluctuation functions $F_{q}(s)$, with a crossover time scale $\delta$ of approximately

$56110^{2}$ seconds. Examination of the generalized Hurst exponents shows that these two scaling

562 regimes correspond to persistent and anti-persistent dynamics for scales below and above the 563 crossover time scale, with the strength of multifractality differing between these two regimes. 
564 When environmental temperature $T_{a}$ is decreased below the $T N Z$, the observed pattern of

565 multifractal, anti-persistent long-range correlations over longer time scales does not vary a great

566 deal. On the other hand, over short scales, the persistent long-range correlations transition from a

567 weakly multifractal to a monofractal distribution. We now discuss these results

569 The first aspect we discuss is the robustness of the rather complex long-correlation structure

570 observed for our data. While previous analysis of $\mathrm{VO}_{2}$ have reported long-range persistent $1 / f^{\beta}$

571 fluctuations, described by a single dominant monofractal scaling exponent (Chaui-Berlinck et al.

572 2002a; Chaui-Berlinck et al. 2002b), we show here that that $\mathrm{VO}_{2}$ fluctuations of different

573 magnitudes are clustered throughout the experimental time series with varying types of long-

574 range correlation, depending on the time scale analyzed. Thus, $r\left(\mathrm{VO}_{2}\right)$ is a multifractal self-affine

575 signal. This suggests that the feedback control mechanisms underlying rapid changes in energy

576 consumption involve strongly non-linear dynamic processes. Both the observed multifractal

577 exponent spectra and the scaling crossover differ from those observed under a random linear

578 transformation in the frequency domain (Kantelhardt 2011; Schreiber \& Schmitz 1996; Schreiber

$579 \&$ Schmitz 2000). This indicates that the observed multifractality of $r\left(\mathrm{VO}_{2}\right)$ is a robust property

580 of metabolic rate. The existence of this long-range correlation structure indicates the potential for

581 plastic dynamic responses to thermal stress (Goldberger et al. 2002; Ivanov et al. 2007). In this

582 regard, the existence of a crossover, with two characteristic long-range correlation signatures

583 may be related to the dynamics of both $\mathrm{VO}_{2}$ and $r\left(\mathrm{VO}_{2}\right)$. As we have shown for data within the

$584 \mathrm{TNZ}$ (see Figure 1), $\mathrm{VO}_{2}$ time series may show periods of higher energy consumption

585 interspersed with periods of lower energy use (Figure 1b). These periods present particularly 
586 different patterns of $\mathrm{VO}_{2}$ changes, which are reflected in the pattern of $r\left(\mathrm{VO}_{2}\right)$ fluctuations. Thus,

587 higher average energy uses (larger mean $\mathrm{VO}_{2}$ values) are associated with less variable values of $588 r\left(\mathrm{VO}_{2}\right)$, in agreement with observed results for inter-specific scaling of $r\left(\mathrm{VO}_{2}\right)$ across different

589 vertebrate species (Labra et al. 2007), as well as in diverse complex systems (see references in 590 Labra et al. 2007). Examination of $r\left(\mathrm{VO}_{2}\right)$ data using different approaches Fourier power spectra,

591 DFA and MFDFA reveal that small-scale and larger scales present different scaling 592 relationships. The first two methods agree qualitatively with the pattern shown by the MF-DFA $593 F_{q}(s)$ fluctuation functions. It is important point to out that that in all series, the scaling crossover 594 was observed regardless of the de-trending polynomial order used in MF-DFA. On the other 595 hand, the type of long-range correlation structure identified was also robust. When data were 596 analysed using MF-DFA using $2^{\text {nd }}$ and $3^{\text {rd }}$ order de-trending polynomials, the scaling regime for 597 smaller time scales is observed to be either weakly multifractal or monofractal across most 598 temperatures, while the second scaling regime is found to be multifractal for all three de-trending 599 orders used in MF-DFA. For the second scaling regime, corresponding to larger time scales, the 600 broadest singularity spectra are observed for $15^{\circ} \mathrm{C}$ and $20^{\circ} \mathrm{C}$, with either $\alpha_{\max } \approx 0.5$ for first de601 trending order MF-DFA, or $0.5>\alpha_{\max }>1.0$ for $2^{\text {nd }}$ and $3^{\text {rd }}$ de-trending order MF-DFA.

602

603 The second aspect we discuss is the possible explanations for the qualitative changes 604 observed in the long-range correlation structure in the vicinity of $15^{\circ} \mathrm{C}$, as well as their potential 605 significance. Metabolic rate changes are central for the control of $T b$ in endotherms (Chaui606 Berlinck et al. 2005; Karasov \& Rio 2007). Thus, body temperature in these organisms is 607 regulated through a complex set of processes and feedback relationships involving behavioral, 
608 endocrine, vasomotor and neural processes (Chaui-Berlinck et al. 2005; Karasov \& Rio 2007). A

609 recent review on the thermal physiology of Mus musculus shows that in this species the lower

610 limit of normothermia ranges between 5 and $15^{\circ} \mathrm{C}$ (Gordon 2012). Below these temperatures,

611 thermal homeostasis requires increased $\mathrm{VO}_{2}$, which become nearly twice the BMR. These

612 additional homeostatic requirements may be offset with different thermoregulation strategies that

613 include behavioral, postural and physiological adjustments, all of which carry with them

614 increased energetic costs. Over longer periods of time, these energetic requirements may not be

615 met without resorting to alternative physiological strategies such as torpor (Gordon 2012).

616 Interestingly, individuals in our measurements did not reach the torpor stage, resorting only to

617 individual huddling within the measurement chamber. Studies on thermoregulatory behavior

618 have shown that small mammals such as lab mice form groups by huddling together as a

619 behavioral thermoregulatory response to temperature challenges (Canals et al. 1997; Canals et al.

620 1998). Interestingly, this behavioral response behaves as a system with a continuous (second-

621 order) phase transition, with a critical environmental temperature value found between $16^{\circ} \mathrm{C}$ and

$62220^{\circ} \mathrm{C}$ (Canals \& Bozinovic 2011). For low temperatures, individuals spontaneously aggregate,

623 forming groups with a higher fractal dimension and a lower mass-specific metabolic rate. This

624 change in behavior occurs in the same temperature range where we have observed maximal

625 values for the degree of multifractality, supporting the idea that different physiological regimes

626 may occur above and below this temperature range. Hence, future work could examine the long-

627 range correlation properties of $\mathrm{VO}_{2}$ fluctuations under different strategies such as torpor or group

628 huddling, in order to determine whether the degree of multifractality decreases below that

629 observed at $0^{\circ} \mathrm{C}$, giving rise either to monofractal scaling or to the loss of fractal 630 autocorrelations. 
631 A third point we discuss is the biological significance of these results. As mentioned earlier,

632 whole-body metabolic rate is an emergent phenomenon, resulting from microscopic interactions

633 with a large number of degrees of freedom and a complex set of opposing feedback mechanisms

634 acting at different time scales (Bozinovic 1992; Chaui-Berlinck et al. 2005). In this regard, the

635 multifractal nature of metabolic rate highlights the complex and non-linear nature of the multiple

636 feedback loops involved in the maintenance of physiological homeostasis (Chaui-Berlinck et al.

637 2005; Darveau et al. 2002; Hochachka et al. 2003). The existence of multifractality in metabolic

638 rate fluctuations has several interesting implications, particularly regarding the sensitivity to

639 initial conditions. In general, multifractal dynamics are generated by non-linear recursive

640 processes, which show different scaling or fractal properties depending on the initial conditions

641 or on the particular history of external disturbances to the system (Kantelhardt 2011). As a result,

642 the observed singularities and scaling exponents of multifractal time series can change in time,

643 leading to the presence of local abrupt shifts in the dynamics of these systems (Kantelhardt

644 2011). In addition, these singularities are associated with the presence of both extreme events

645 and fat tailed power law distributions, which have been shown to be a universal feature of

646 metabolic rate across different vertebrate species (Labra et al. 2007). Despite the seemingly

647 irregular unpredictable nature of metabolic rate fluctuations, our results show that they have a

648 characteristic long-range correlation structure. Although in many applications the proximal

649 mechanistic causes of observed fractality or multifractality have not been elucidated (Kantelhardt

650 2011), the fact remains that multifractal processes such as $r\left(\mathrm{VO}_{2}\right)$ are completely different from

651 simple linear random fluctuations. This opens an interesting scenario regarding the potential use

652 of multifractal properties as either a diagnostic tool or as baseline to determine animal response

653 to environmental stress. This improved characterization may also eventually allow the modeling 
654 the dynamics and projection of the likelihood of extreme events or prediction of future behavior 655 (Kantelhardt 2011). This may complement the empirical estimates of metabolic rate, which 656 typically correspond to the average value of $\mathrm{VO}_{2}$ registered in a small section of the time series 657 under specific environmental conditions (Lighton 2008). Similarly, measurements of the rate of $658 \mathrm{VO}_{2}$ under the maximum sustainable rate of exercise (i.e. maximal metabolic rate) have been 659 shown to be mostly a function of aerobic capacity of the muscle mass (Weibel et al. 2004). In the 660 light of our results, it seems reasonable to expect that $\mathrm{VO}_{2}$ fluctuations under conditions of 661 maximum sustainable exercise would also show multifractal long-term correlations as well as 662 power law distributed fluctuations.

663

664 In addition to the physiological significance of long-range multifractal correlations of $r\left(\mathrm{VO}_{2}\right)$, 665 a related aspect pertains the taxonomic and systemic generality and significance of our results. It 666 is relevant to discuss whether these observed patterns are expected to hold true for all 667 endothermic species. While previous work on $r\left(\mathrm{VO}_{2}\right)$ has reported a universal probability 668 distribution function across different vertebrate species (Labra et al. 2007), no systematic 669 comparative assessment has been carried out to determine if the long-range correlation structure 670 may hold true for different endothermic species, be these birds or mammals. A particularly 671 interesting aspect of such comparisons would be to examine the role of individual body size. Our 672 work was carried out using a small endothermic species, the lab mouse. Analysis of a theoretical 673 model of body temperature control by shifts in metabolic rate has suggested that the rate of heat

674 loss and the capacity to rapidly increase metabolic output may lead to non-equilibrium between 675 metabolic rate and body temperature in micro-endotherms (such as hummingbirds and small 
676 mice), resulting in non-random $1 / f^{\beta}$ persistent oscillations of $\mathrm{VO}_{2}$, even within the $T N Z$ (Chaui-

677 Berlinck et al. 2002a). Our results indicate that $\mathrm{VO}_{2}$ are not only long-range correlated, but that

678 have a complex multifractal structure, which indicates that the model of Chaui-Berlinck et al.

679 (2002a) yields predictions that are at least qualitatively correct. Interestingly, this theoretical

680 model also predicts that larger endotherms such as the rat may not exhibit similar complex

681 oscillations, due to a dynamic equilibrium between metabolic rate and body temperature, given

682 the smaller surface area-volume ratio. If correct, this model predicts the absence of long-range

683 correlated $r\left(\mathrm{VO}_{2}\right)$ oscillations for larger endotherms, with multifractal dynamics being found

684 only in micro-endotherms, regardless of whether they are mammals or birds. Whether a

685 threshold body size may be identified below which multifractality may be observed would

686 indicate the onset of a highly nonlinear configuration of control processes acting in the regulation

687 of body temperature. The alternative outcome would be that multifractal long-range correlations

688 also hold true for larger endotherms. This alternative scenario would indicate that a more

689 detailed model analysis is required to account for the processes affecting metabolic rate 690 oscillations.

\section{General Conclusion}

692 While an increasing number of authors have pointed out the complex nature of physiological 693 processes (Burggren \& Monticino 2005; Spicer \& Gaston 2009), an emerging research question 694 is what are the consequences and implications of physiological complexity for the homeostatic 695 adaptive capability of animals, particularly on a scenario of global climate change. In addition to 696 considering the potential role of organism body size, it is important to determine whether the 697 observed multifractal correlation structure is a general trait of all endotherm taxa, or if it is a 
698 characteristic trait of mammals as a lineage. Comparative experimental studies may help to

699 untangle the relative importance of body size and taxonomic inertia in the emergence of

700 multifractality. A related question is whether ectotherms do present any long-range correlation

701 structure in their metabolic rate dynamics. If complexity is an emergent characteristic arising

702 from the different thermal control feedback loops, then multifractality should be absent in

703 metabolic rate dynamics of reptiles or amphibians. The goal of such studies would be to allow

704 the assessment of the relative importance of universal emergent statistical behaviour and

705 phylogenetic inertia in morphological and physiological traits that may give rise to complex

706 metabolic rate fluctuations. Again, the use of a comparative, controlled experimental approach

707 may allow careful examination of the relationships between the complexity of metabolic rate

708 dynamics and the origins of endothermy.

709

710

711

712

713 Our results show that the dynamic response of the metabolic machinery in a model mammal

714 species facing thermal challenge do not reduce themselves to the linear variance response

715 expected, evidencing in addition that this response is regulated by environmental history

716 experienced of individual. In this regards, the humped shape observed from the relationship

717 between complexity level of $\mathrm{VO}_{2}$ and decrease of temperature agree with a limit at the

718 physiological capability to control of body temperature. Future work in this area may focus on 
719 experimental explorations of the physiological basis of long-term correlations and multifractality

720 of $\mathrm{VO}_{2}$ fluctuations. For example, such work may examine the relative importance of different

721 control mechanisms regulating the rate of oxygen uptake as part of a hierarchical cascade of

722 feedback loops that lead to multifractality.

723

724 Acknowledgments

725 We thank F. Boher and S. Clavijo for their assistance during the development of these

726 experiments. FAL thanks C. Huerta and E. Labra for their continued support.

727

728 
729

730

731

732

733

734

735

736

737

738

739

740

741

742

743

744

745

746

\section{References}

Angilletta MJ. 2006. Estimating and comparing thermal performance curves. Journal of Thermal Biology 31:541-545.

Ashkenazy Y, Baker DR, Gildor H, and Havlin S. 2003. Nonlinearity and multifractality of climate change in the past 420,000 years. Geophys Res Lett 30:2146. $10.1029 / 2003 \mathrm{~g} 1018099$

Bassingthwaighte, J. B., \& Raymond, G. M. (1994). Evaluating rescaled range analysis for time series. Annals of biomedical engineering, 22(4), 432-444.

Bahar, S., Kantelhardt, J. W., Neiman, A., Rego, H. H. A., Russell, D. F., Wilkens, L., ... \& Moss, F. (2001). Long-range temporal anti-correlations in paddlefish electroreceptors. EPL (Europhysics Letters), 56(3), 454.

Billat, V. L., Wesfreid, E., Kapfer, C., Koralsztein, J. P., \& Meyer, Y. (2006). Nonlinear dynamics of heart rate and oxygen uptake in exhaustive 10,000 m runs: influence of constant vs. freely paced. The Journal of Physiological Sciences, 56(1), 103-111.

Bozinovic F. 1992. Scaling of basal and maximum metabolic rate in rodents and the aerobic capacity model for the evolution of endothermy. Physiological Zoology 65:921-932.

Bozinovic F, and Rosenmann M. 1988. Comparative energetics of South American cricetid rodents. Comparative Biochemistry and Physiology Part A, Physiology 91:195-202.

747 Bunde A, and Lennartz S. 2012. Long-term correlations in earth sciences. Acta Geophysica 
60:562-588.

749 Burggren WW, and Monticino AG. 2005. Assessing physiological complexity. Journal of Experimental Biology 208:3221-3232. 10.1242/jeb.01762

751 Canals M, and Bozinovic F. 2011. Huddling Behavior as Critical Phase Transition Triggered by Low Temperatures. Complexity 17:35-43. 10.1002/cplx.20370

753

Canals M, Rosenmann M, and Bozinovic F. 1997. Geometrical aspects of the energetic effectiveness of huddling in small mammals. Acta Theriologica 42:321-328.

Canals M, Rosenmann M, Novoa FF, and Bozinovic F. 1998. Modulating factors of the energetic effectiveness of huddling in small mammals. Acta Theriologica 43:337-348.

Chaui-Berlinck JG, Bicudo J, Monteiro LHA, and Navas CA. 2002a. Oscillatory pattern in oxygen consumption of hummingbirds. Journal of Thermal Biology 27:371-379. Pii $\underline{\mathrm{s} 0306-4565(02) 00005-010.1016 / \mathrm{s} 0306-4565(02) 00005-0}$

Chaui-Berlinck JG, Monteiro LHA, Navas CA, and Bicudo J. 2002b. Temperature effects on energy metabolism: a dynamic system analysis. Proceedings of the Royal Society B: Biological Sciences 269:15-19. 10.1098/rspb.2001.1845

Chaui-Berlinck JG, Navas CA, Monteiro LHA, and Bicudo J. 2005. Control of metabolic rate is a hidden variable in the allometric scaling of homeotherms. Journal of Experimental Biology 208:1709-1716. 10.1242/jeb.01421

Costa M, Ghiran I, Peng CK, Nicholson-Weller A, and Goldberger AL. 2008. Complex 
dynamics of human red blood cell flickering: alterations with in vivo aging. Physical Review E, Statistical, nonlinear, and soft matter physics 78:020901.

769 Costa M, Goldberger AL, and Peng CK. 2002. Multiscale entropy analysis of complex 770 physiologic time series. Physical Review Letters 89:068102.

771

772

773

774

775

776

777

778

779

780

781

782

783

Feder J. 1988. Fractals: Plenum Press.

784 785 $10.1038 / 417166 \mathrm{a}$ 10.1152/japplphysiol.90757.2008 e1001089. Archives, 439, 403-415. A 238:244-252.

Darveau CA, Suarez RK, Andrews RD, and Hochachka PW. 2002. Allometric cascade as a unifying principle of body mass effects on metabolism. Nature 417:166-170.

Delignières D, and Torre K. 2009. Fractal dynamics of human gait: a reassessment of the 1996 data of Hausdorff et al. Journal of Applied Physiology 106:1272-1279.

Delignières, D., Torre, K., \& Bernard, P. L. (2011). Transition from persistent to anti-persistent correlations in postural sway indicates velocity-based control. PLoS Comput Biol, 7(2),

Eke, A., Herman, P., Bassingthwaighte, J. B., Raymond, G. M., Percival, D. B., Cannon, M., et al. (2000). Physiological time series: Distinguishing fractal noises from motions. Pflügers

Feldman DP, and Crutchfield JP. 1998. Measures of statistical complexity: Why? Physics Letters 
786 Glass L. 2001. Synchronization and rhythmic processes in physiology. Nature 410:277-284. 787 $10.1038 / 35065745$

788 Goldberger AL, Rigney DR, West BJ. Chaos and fractals in human physiology. Sci Am 789 1990;262:40-9.

790 791

792 793

794 795

Goldberger AL. Non-linear dynamics for clinicians: chaos theory, fractals, and complexity at the bedside. Lancet 1996;347:1312-4.

Goldberger AL, Amaral LAN, Hausdorff JM, Ivanov PC, Peng CK, and Stanley HE. 2002. Fractal dynamics in physiology: Alterations with disease and aging. Proceedings of the National Academy of Sciences of the United States of America 99:2466-2472. 10.1073/pnas.012579499

Goldberger AL, and West BJ. 1987. Fractals in physiology and medicine. The Yale journal of biology and medicine 60:421.

Gordon CJ. 2012. Thermal physiology of laboratory mice: Defining thermoneutrality. Journal of Thermal Biology 37:654-685. http://dx.doi.org/10.1016/j.jtherbio.2012.08.004

Hausdorff JM, Ashkenazy Y, Peng CK, Ivanov PC, Stanley HE, and Goldberger AL. 2001. When human walking becomes random walking: fractal analysis and modeling of gait rhythm fluctuations. Physica A 302:138-147. 10.1016/s0378-4371(01)00460-5

Hochachka PW, Darveau CA, Andrews RD, and Suarez RK. 2003. Allometric cascade: a model for resolving body mass effects on metabolism. Comparative biochemistry and physiology Part A, Molecular \& integrative physiology 134:675-691. 10.1016/s1095- 
807 Hu K, Ivanov PC, Chen Z, Hilton MF, Stanley HE, and Shea SA. 2004. Non-random fluctuations 808 and multi-scale dynamics regulation of human activity. Physica A 337:307-318. 809 10.1016/j.physa.2004.01.042

810 Humeau A, Buard B, Chapeau-Blondeau F, Rousseau D, Mahe G, and Abraham P. 2009. 811 Multifractal analysis of central (electrocardiography) and peripheral (laser Doppler 812 flowmetry) cardiovascular time series from healthy human subjects. Physiological Measurement 30:617-629. 10.1088/0967-3334/30/7/007

814 Hurst HE. 1951. Long-term storage capacity of reservoirs. T Am Soc CIiv Eng 116:770-799.

815 Ihlen, EA. 2012. Introduction to multifractal detrended fluctuation analysis in Matlab. Frontiers in Physiology, 3:141.x1-141.18 . 10.3389/fphys.2012.00141

817 Ivanov PC, Amaral LAN, Goldberger AL, Havlin S, Rosenblum MG, Struzik ZR, and Stanley

818 HE. 1999. Multifractality in human heartbeat dynamics. Nature 399:461-465. $10.1038 / 20924$

Ivanov PC, Hu K, Hilton MF, Shea SA, and Stanley HE. 2007. Endogenous circadian rhythm in human motor activity uncoupled from circadian influences on cardiac dynamics. Proceedings of the National Academy of Sciences of the United States of America Complexity and Dynamical Systems. New York: Springer, 463-487. 
826 Kantelhardt JW, Koscielny-Bunde E, Rybski D, Braun P, Bunde A, and Havlin S. 2006. Long-

827

828

829

830

831

832

833

834

835

836

837

838

839

840

841

842

843

844

845

term persistence and multifractality of precipitation and river runoff records. Journal of geophysical research Atmospheres : JGR 111. D01106

$\underline{10.1029 / 2005 \mathrm{jd} 005881}$

Kantelhardt JW, Rybski D, Zschiegner SA, Braun P, Koscielny-Bunde E, Livina V, Havlin S, and Bunde A. 2003. Multifractality of river runoff and precipitation: comparison of fluctuation analysis and wavelet methods. Physica A 330:240-245. 10.1016/j.physa.2003.08.019

Kantelhardt JW, Zschiegner SA, Koscielny-Bunde E, Havlin S, Bunde A, and Stanley HE. 2002. Multifractal detrended fluctuation analysis of nonstationary time series. Physica $A$ 316:87-114. Pii s0378-4371(02)01383-3

$\underline{10.1016 / \mathrm{s} 0378-4371(02) 01383-3}$

Karasov WH, and Rio CM. 2007. Physiological ecology: how animals process energy, nutrients, and toxins: Princeton University Press.

Koscielny-Bunde E, Kantelhardt JW, Braun P, Bunde A, and Havlin S. 2006. Long-term persistence and multifractality of river runoff records: Detrended fluctuation studies. Journal of Hydrology 322:120-137. 10.1016/i.jhydro1.2005.03.004

Labra FA, Marquet PA, and Bozinovic F. 2007. Scaling metabolic rate fluctuations. Proceedings of the National Academy of Sciences of the United States of America 104:10900-10903. 10.1073/pnas.0704108104 
846 Lighton JRB. 2008. Measuring metabolic rates: a manual for scientists: Oxford University 847 Press.

848 Lipsitz LA. 2004. Physiological complexity, aging, and the path to frailty. Science of aging 849 knowledge environment : SAGE KE 2004:pe16. 10.1126/sageke.2004.16.pe16

850 Ludescher J, Bogachev MI, Kantelhardt JW, Schumann AY, and Bunde A. 2011. On spurious 851

852 and corrupted multifractality: The effects of additive noise, short-term memory and periodic trends. Physica A 390:2480-2490. 10.1016/j.physa.2011.03.008

853 Mantegna RN, and Stanley HE. 1997. Econophysics: Scaling and its breakdown in finance. 854 Journal of Statistical Physics 89:469-479. 10.1007/bf02770777

855 Mantegna RN, and Stanley HE. 2000. An Introduction to Econophysics: Correlations and 856 Complexity in Finance: Cambridge University Press.

McNab BK. 2002. The physiological ecology of vertebrates: a view from energetics: Cornell $858 \quad$ University Press.

859 Muggeo, V. 2003. Estimating regression models with un-known break-points. Statistics in $860 \quad$ Medicine, 22: 3055-3071.

861 Oswiecimka P, Kwapien J, and Drozdz S. 2006. Wavelet versus detrended fluctuation analysis of 862 multifractal structures. Physical Review E, Statistical, nonlinear, and soft matter physics 863 74. $\underline{01610310.1103 / \text { PhysRevE.74.016103 }}$

864 Peng CK, Buldyrev SV, Goldberger AL, Havlin S, Mantegna RN, Simons M, and Stanley HE. 
867 Peng CK, Havlin S, Stanley HE, and Goldberger AL. 1995b. Quantification of scaling exponents 868 and crossover phenomena in nonstationary heartbeat time-series. Chaos 5:82-87.

869 $\underline{10.1063 / 1.166141}$

Peng CK, Mietus J, Hausdorff JM, Havlin S, Stanley HE, and Goldberger AL. 1993. Long-range anticorrelations and non-gaussian behavior of the heartbeat. Physical Review Letters 70:1343-1346. 10.1103/PhysRevLett.70.1343

Peng CK, Mietus JE, Liu Y, Lee C, Hausdorff JM, Stanley HE, Goldberger AL, and Lipsitz LA. 2002. Quantifying fractal dynamics of human respiration: age and gender effects. Annals of Biomedical Engineering 30:683-692.

876 Pincus SM. 1991. Approximate entropy as a measure of system complexity. Proceedings of the National Academy of Sciences 88:2297-2301.

878 R Core Team (2014). R: A language and environment for statistical computing. R Foundation for 879 Statistical Computing, Vienna, Austria. URL http://www.R-project.org/.

Rezek IA, and Roberts SJ. 1998. Stochastic complexity measures for physiological signal 881 analysis. IEEE Transactions on Biomedical Engineering 45:1186-1191.

882 Richman JS, and Moorman JR. 2000. Physiological time-series analysis using approximate 883 entropy and sample entropy. American Journal of Physiology-Heart and Circulatory 884 Physiology 278:H2039-H2049. 
885 Schaefer, A., Brach, J. S., Perera, S., \& Sejdić, E. (2014). A comparative analysis of spectral 886 exponent estimation techniques for $1 / \mathrm{f}^{\beta}$ processes with applications to the analysis of 887 stride interval time series. Journal of neuroscience methods, 222, 118-130.

888 Schreiber T, and Schmitz A. 1996. Improved surrogate data for nonlinearity tests. Physical 889 Review Letters 77:635-638. 10.1103/PhysRevLett.77.635

890 Schreiber T, and Schmitz A. 2000. Surrogate time series. Physica D 142:346-382. 891 $10.1016 / \mathrm{s} 0167-2789(00) 00043-9$

892 Spicer J, and Gaston K. 2009. Physiological Diversity: Ecological Implications: Wiley. com.

893 Suki B, Alencar AM, Frey U, Ivanov PC, Buldyrev SV, Majumdar A, Stanley HE, Dawson CA, 894 Krenz GS, and Mishima M. 2003. Fluctuations, noise and scaling in the cardio895 pulmonary system. Fluct N Lett 3:R1-R25. 10.1142/s0219477503001142

896 Weibel ER, Bacigalupe LD, Schmitt B, and Hoppeler H. 2004. Allometric scaling of maximal 897 898 metabolic rate in mammals: muscle aerobic capacity as determinant factor. Respiratory physiology \& neurobiology 140:115-132. 10.1016/j.resp.2004.01.006

899

900

901

902

West BJ, Bassingthwaighte JB, and Liebovitch LS. 1994. Fractal physiology: Oxford University Press.

West BJ, and Scafetta N. 2003. Nonlinear dynamical model of human gait. Physical review E 67. 051917.10.1103/PhysRevE.67.051917 
$904 \quad$ Modern Physics B, 3(06), 795-819.

905 Witt, A., \& Malamud, B. D. (2013). Quantification of long-range persistence in geophysical time

906 series: conventional and benchmark-based improvement techniques. Surveys in $907 \quad$ Geophysics, 34(5), 541-651.

908

909 


\section{Figure $\mathbf{1}$ (on next page)}

Long-term correlations of metabolic rate fluctuations in Mus musculus.

(a) Average metabolic rates $\left(\mathrm{VO}_{2}\right)$ measured at different ambient temperatures. Average values \pm standard errors are shown with open circles and error bars. Straight line shows calculated thermal conductance, while the humped curve corresponds to a fitted three parameter Gaussian function $\left(g(x)=a * \exp \left(-.5^{*}((x-x 0) / b)^{\wedge} 2\right)\right)$. (b) Metabolic rate $\left(V_{2}\right)$ time series shown for a representative individual measured at $30^{\circ} \mathrm{C}$ for $13 / 4$ hours at $1(\mathrm{~s})$ intervals. Note the irregular, nonstationary dynamics, despite thermo neutral ambient temperature. (c) Observed $\mathrm{VO}_{2}$ fluctuations $r(\mathrm{VO} 2)=\log 10\left[\mathrm{VO}_{2}(t+1) / \mathrm{VO}_{2}(t)\right]$ time series for data in (b). Note the clustering of broad and narrow fluctuations. (d) Randomized $r\left(\mathrm{VO}_{2}\right)$ values, showing the loss of the clustering of fluctuations. (e) Fourier power spectra for time series in (c) and (d) shown by blue and red lines respectively. A smoothing procedure was applied, which consisted of averaging the spectra for consecutive overlapping segments of 256 data points. Fitted OLS scaling relationships are shown in dotted lines. (f) Detrended fluctuation analyses (DFA) for the two time series shown in (c) and (d). Fluctuation functions for original and shuffled time series in are shown in open and filled circles respectively. Fitted scaling relationships are shown in dashed lines. Note the change in exponent values above $s=100$ for the original time series. 

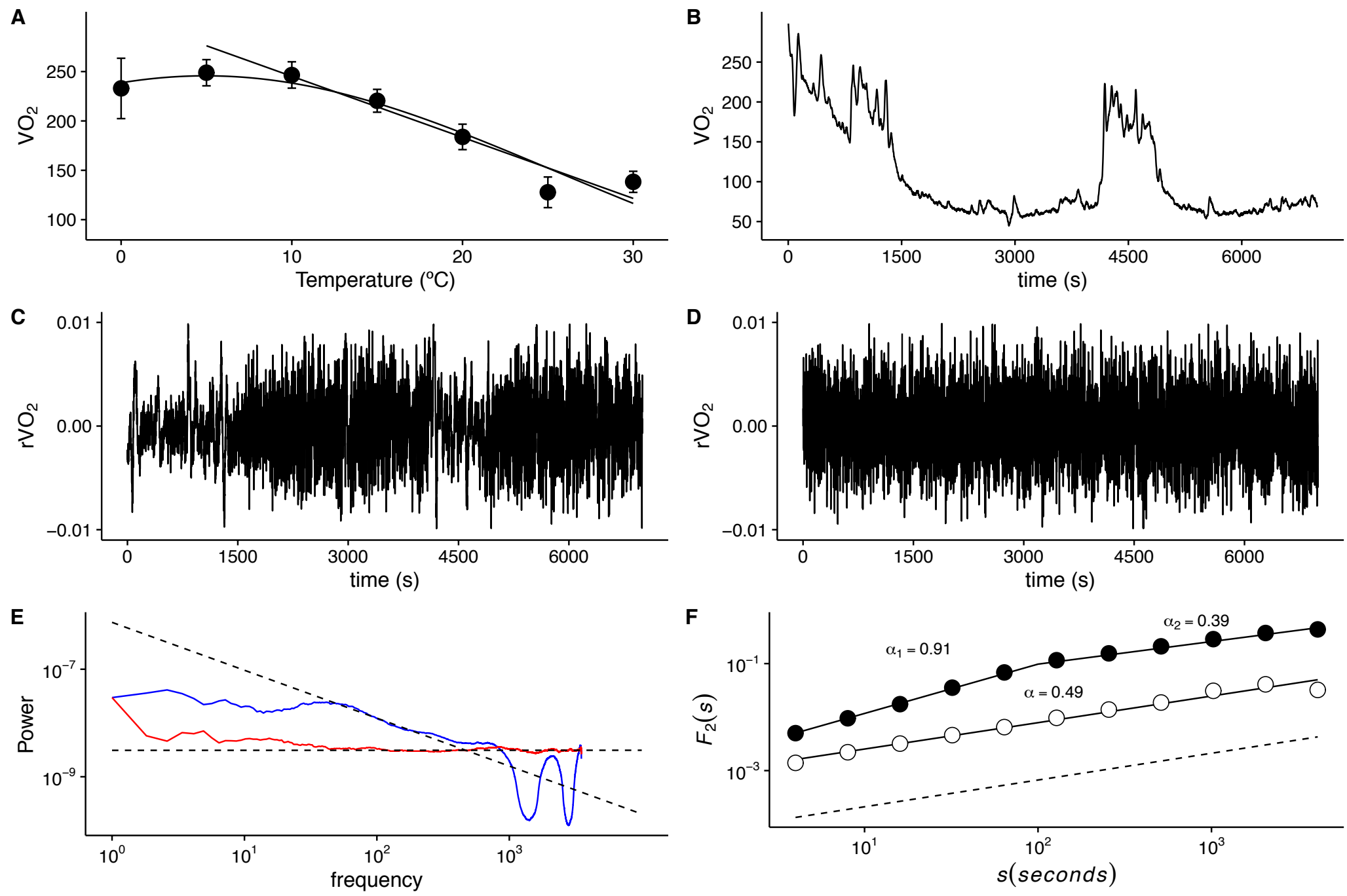


\section{Figure 2 (on next page)}

Temperature effects on root-mean-square fluctuation function of $r\left(\mathrm{VO}_{2}\right)$ in mice.

The figure shows the average $F_{2}(s)$ functions calculated with linear detrending for all mice. Results for the time series studied at $30^{\circ} \mathrm{C}, 20^{\circ} \mathrm{C}, 10^{\circ} \mathrm{C}$ and $0^{\circ} \mathrm{C}$ are shown in the respective columns arranged from left to right. Figures (a) to (d) show the average DFA functions calculated for the $r\left(\mathrm{VO}_{2}\right)$ time series, while figures (e) to (h) show average DFA functions calculated for the AAFT shuffled data. All figures show the DFA root-mean-square fluctuation functions obtained using three different orders of detrending polynomials: linear (open circles), quadratic (open squares) and cubic functions (open triangles). Two scaling regimes can be observed across all temperatures and for all polynomial detrending orders. The first scaling regime spans scales between 8 and $100 \mathrm{~s}$, while the second one spans scales from 100 to $1024 \mathrm{~s}$. All curves have been shifted vertically for clarity. Please note that while only four experimental temperatures are shown, the remaining three temperatures show similar patterns. 

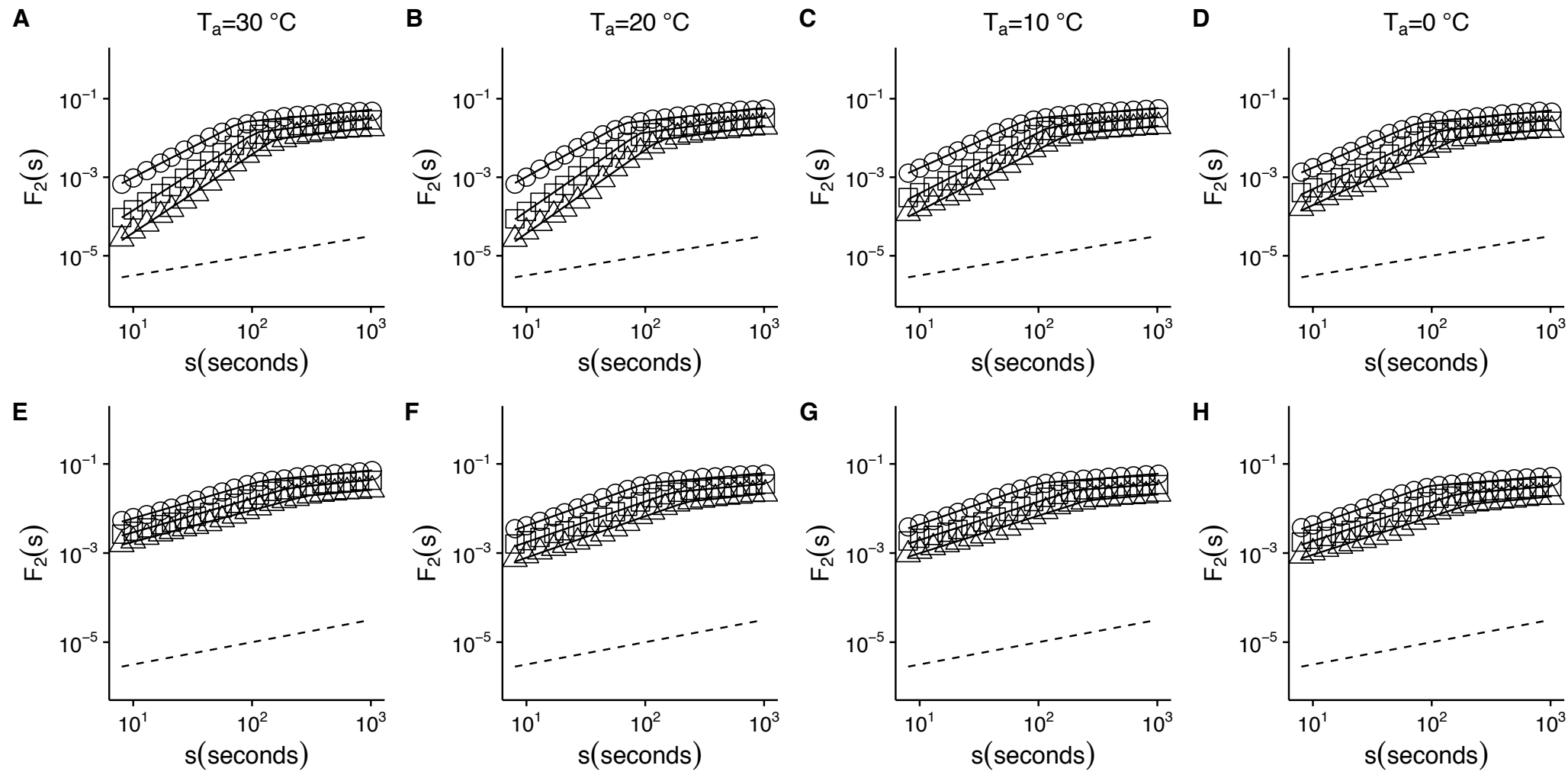
Figure 3 (on next page)

Temperature effects on long range scaling exponent $\alpha$ in metabolic rate fluctuations.

The figure shows the average DFA scaling exponent $\alpha_{D F A}$ calculated as a function of experimental temperature. Average scaling exponents corresponding to exponent for raw $r$ (VO2) data within the $10<s<100$ scaling regime are shown with filled circles, while filled squares show the scaling exponents for the raw $r$ (VO2) data within the $100<s<1024$ scaling regimes are shown with. 

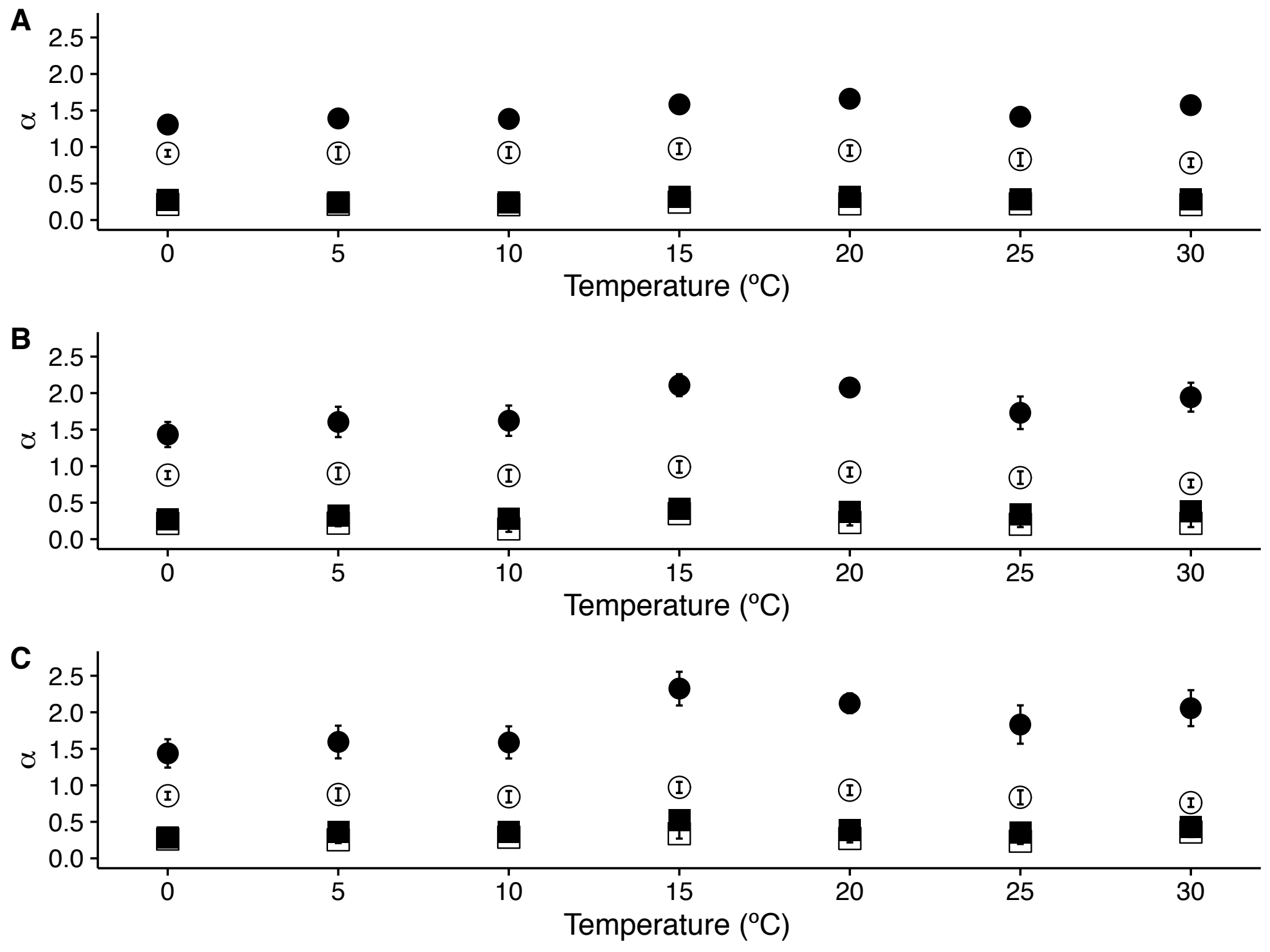
Figure 4(on next page)

Local DFA scaling exponents.

The Figure shows the value of local DFA scaling exponents $\alpha_{D F A}$ for the time series in figures 1c (blue lines) and $1 \mathrm{~d}$ (red lines). Local exponents are calculated with a moving window shifted across the whole time series. Figures (a), (b) and (c) show the results for shifting window widths of 128, 256 and 512 seconds respectively. The heterogeneity of the rate of change in metabolic rate is revealed by the broad range of local scaling exponents $\alpha_{D F A}$, which shows a complex structure in time as opposed to the simpler and more restricted changes in the shuffled time series. 

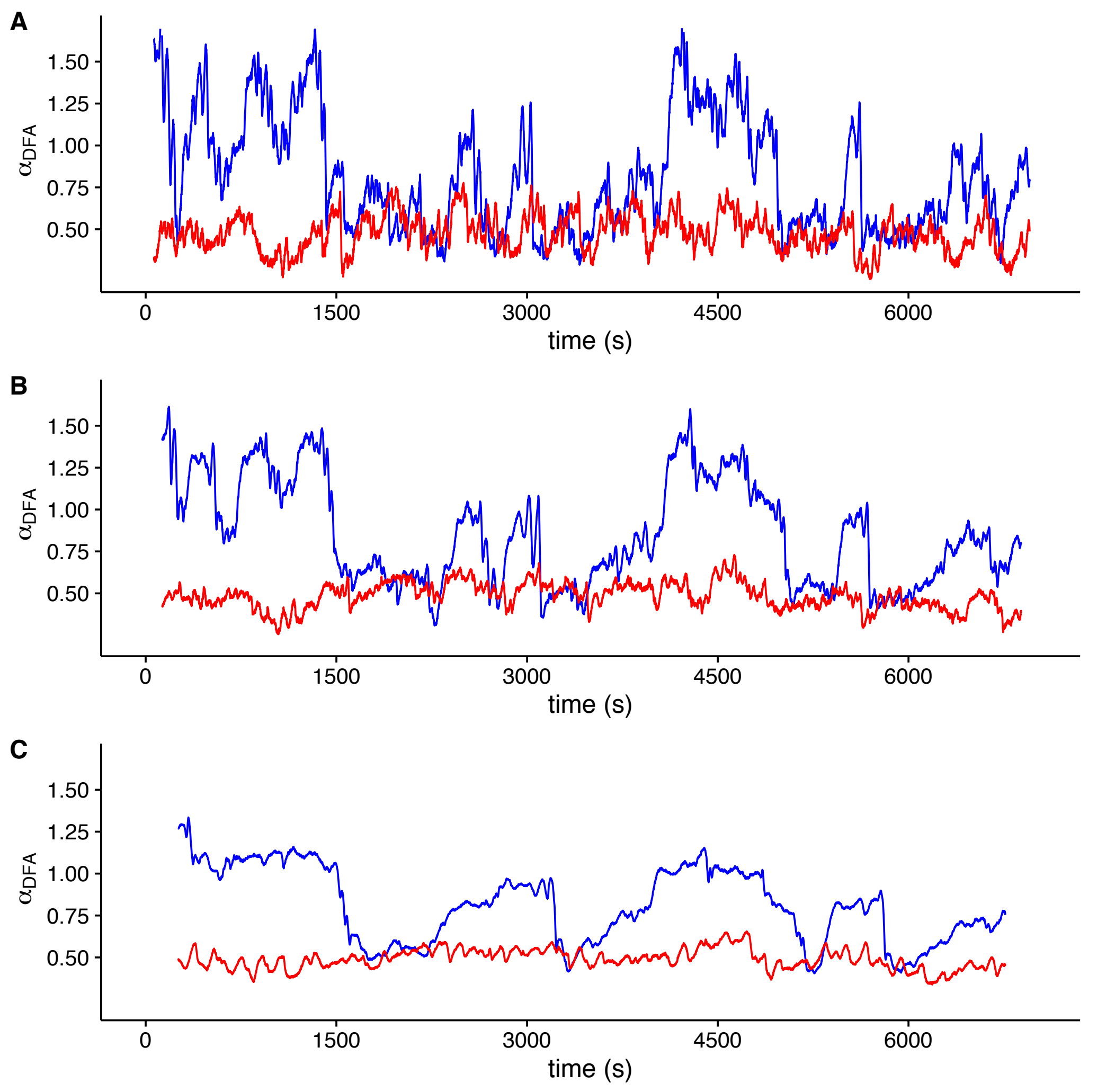


\section{Figure 5 (on next page)}

Temperature effects on generalized fluctuation function of $r\left(\mathrm{VO}_{2}\right)$ in mice.

Figure shows log-log plots of the average generalized fluctuation function $F_{q}(s)$ as a function of time $s$ in $r\left(\right.$ VO2) time series. Columns left to right show the results for $F_{q}(s)$ functions calculated for $30^{\circ} \mathrm{C}, 20^{\circ} \mathrm{C}, 10^{\circ} \mathrm{C}$ and $0^{\circ} \mathrm{C}$ respectively. Figures (a) to (d) show the average $F_{q}(s)$ functions calculated for the $r\left(\mathrm{VO}_{2}\right)$ time series, while figures (e) to (h) show average $F_{q}(s)$ functions calculated for the AAFT shuffled data. Open circles in all figures show the observed $F_{q}(s)$ values for different values of $q$, with $q=8,4,2,1,0,-1,-2,-4$, and -8 (from the top to the bottom). Also shown in black lines are piecewise linear regression fits to the $F_{q}(s)$ functions. Dashed straight lines with slope $h=0.5$ are shown below the data in each figure to allow qualitative comparison with the uncorrelated case. Please note that while only four experimental temperatures are shown, the remaining three temperatures show similar patterns. 

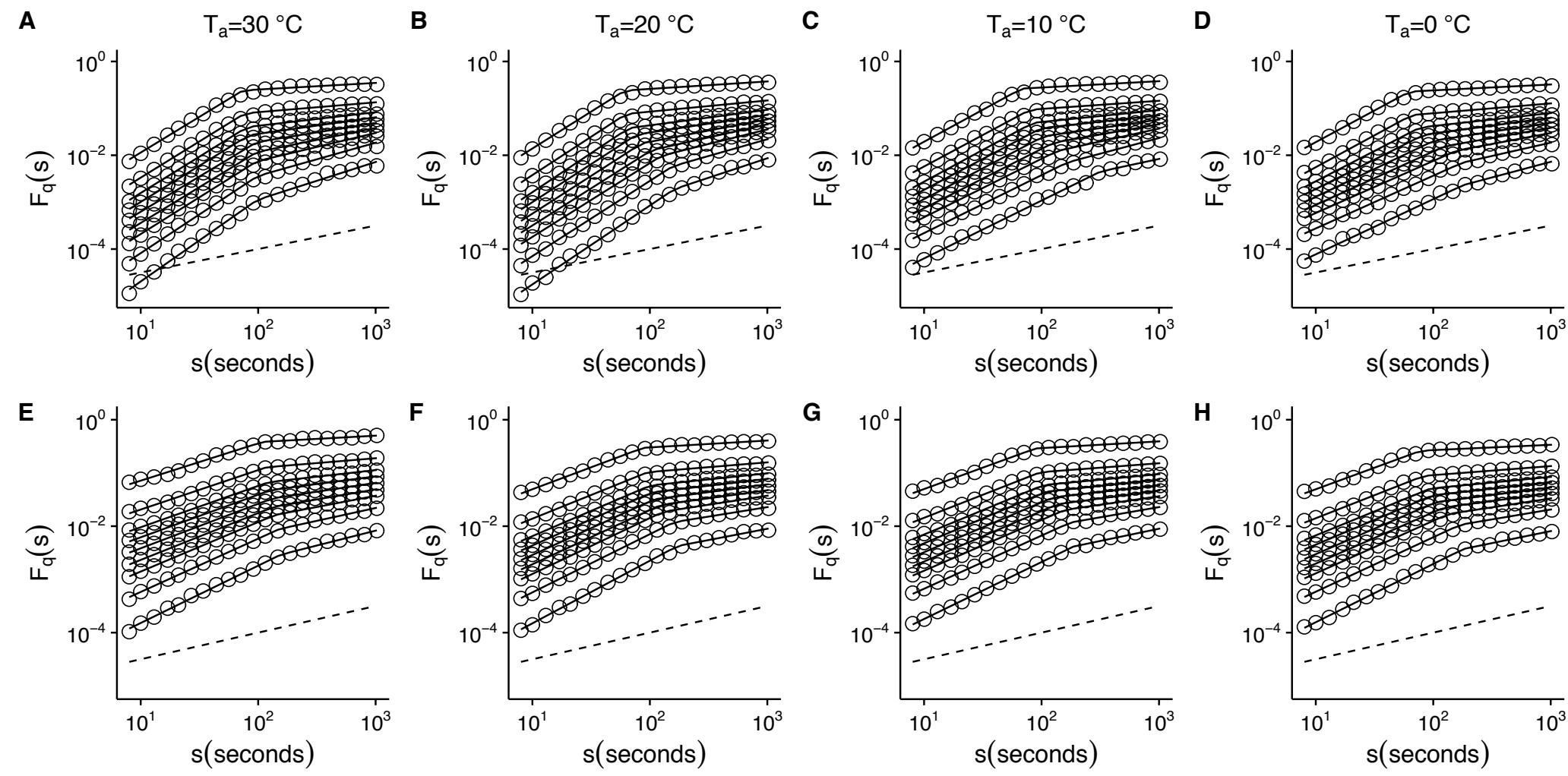


\section{Figure 6 (on next page)}

Multifractal Detrended Fluctuation Analysis of Mus musculus r(VO2) time series across different temperature treatments.

The figure shows the results of the multifractal scaling analysis for all mice studied. Left, central and right hand column show the results for the generalized Hurst exponent spectra $(h(q))$, Renyi exponent spectra $(\tau(q))$ and singularity spectra $(f(\alpha))$. Each figure shows in dashed and continuous black lines the smoothed conditional mean of the different spectra for the first and second scaling regimes respectively. For shuffled data, the smoothed conditional mean of the different spectra for the first and second scaling regimes are shown by dashed and continuous red lines respectively. For figures (c), (f) and (i), the singularity spectra of the first regime corresponds to a single point, shown by a filled circle. The singularity spectra reveal that for temperatures in the range $0^{\circ} \mathrm{C}<\mathrm{T}_{\mathrm{a}}<10^{\circ} \mathrm{C}$ the time scales in the $8<\mathrm{s}<100$ range present a monofractal scaling, while all remaining temperatures show a weak multifractal scaling. All data for the second scaling regime show strong multifractality, which is not completely lost when data are shuffled. 

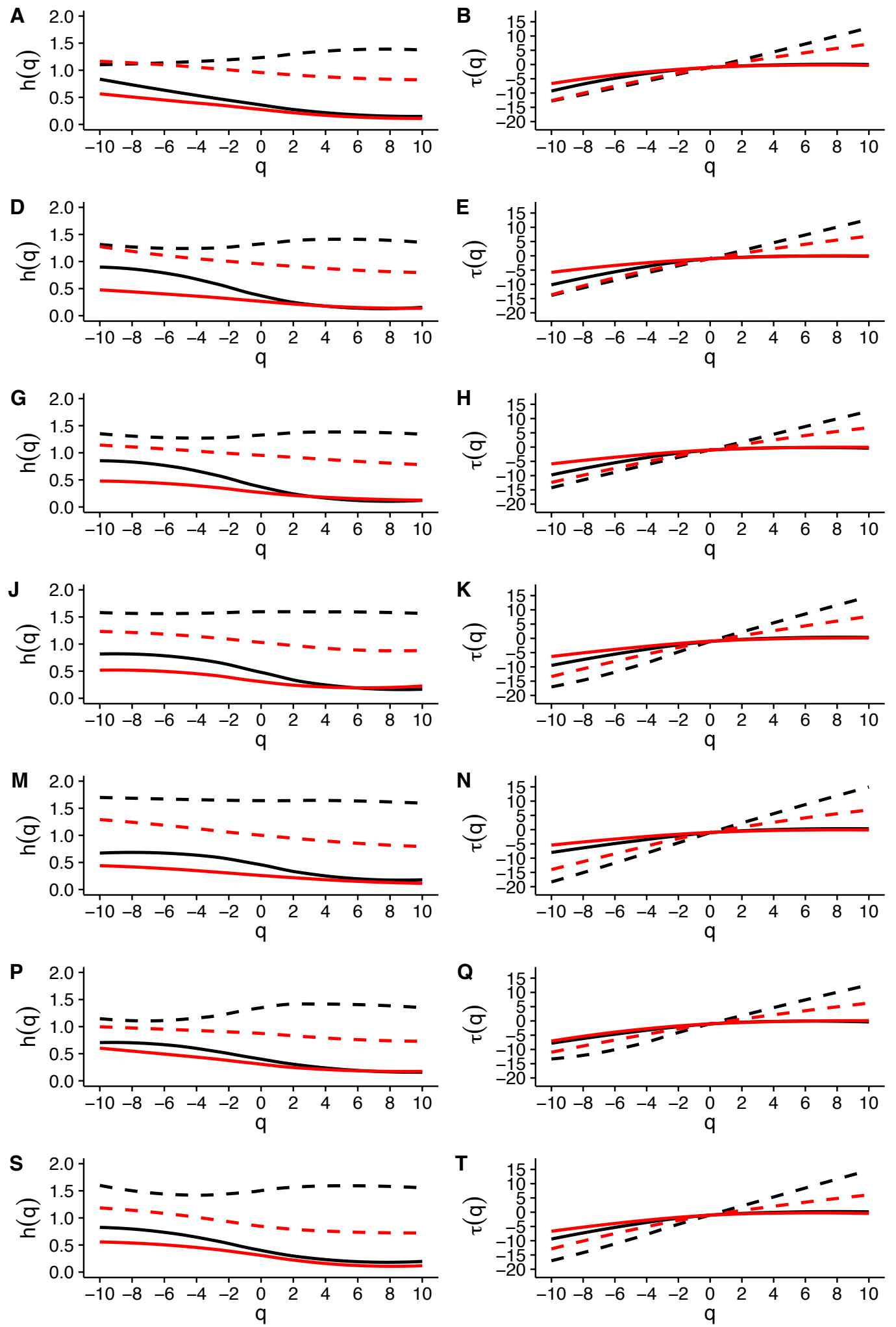
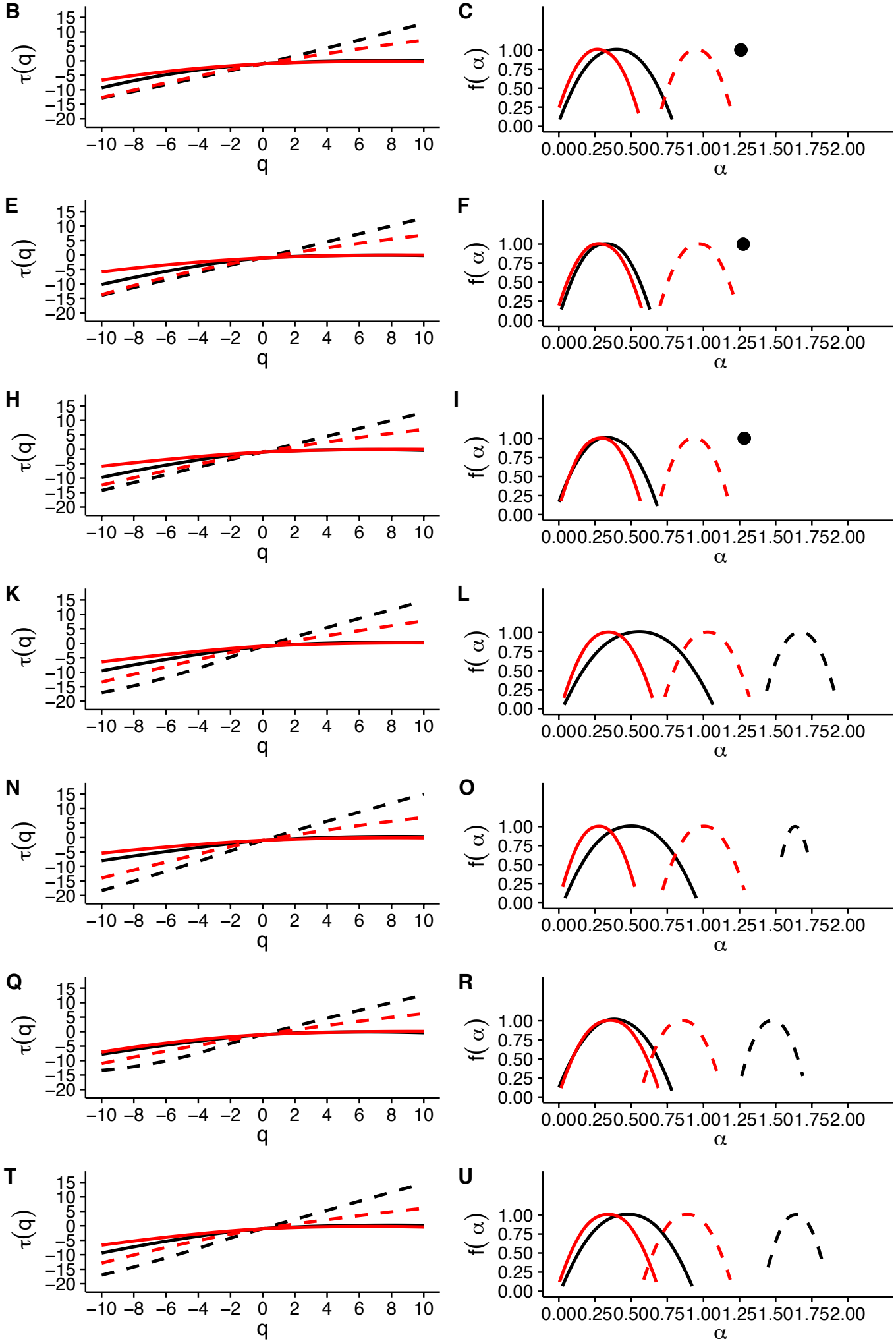
Figure 7 (on next page)

Temperature effects on the strength of multifractality in mice.

The figure shows the average widths $\Delta \alpha$, of the $f(\alpha)$ spectra as a function of environmental temperature $T_{a}$. Left hand, central and right hand columns show the results for linear, quadratic and cubic polynomial de-trending respectively. Figures (a) to (d) show the average $\Delta \alpha$ values calculated for the $r\left(\mathrm{VO}_{2}\right)$ time series, while figures (e) to (h) show the average $\Delta \alpha$ values calculated for the AAFT shuffled data. 

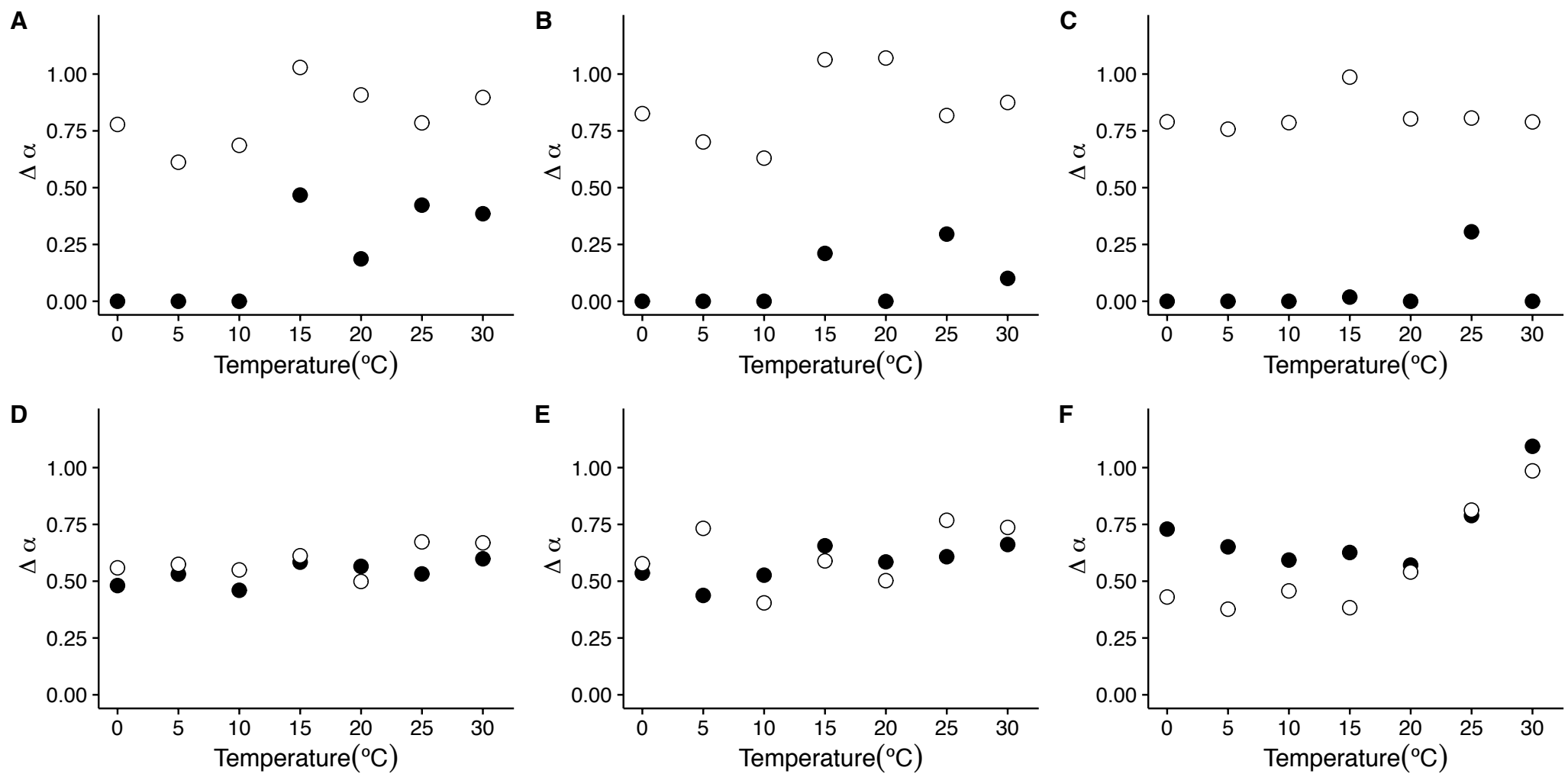


\section{Figure 8 (on next page)}

Temperature effects on the dominant multifractal exponent in mice.

Temperature effects on the dominant multifractal exponent in Mus musculus. The figure shows the average dominant fractal exponent $\alpha_{\text {max }}$ for the different the $f(\alpha)$ spectra as a function of environmental temperature $T_{a}$. The left hand, central and right hand columns show the results for linear, quadratic and cubic polynomial detrending respectively. Figures (a) to (d) show the average $\alpha_{\max }$ values calculated for the $r\left(\mathrm{VO}_{2}\right)$ time series, while figures (e) to (h) show the average $\alpha_{\max }$ values calculated for the AAFT shuffled data. 

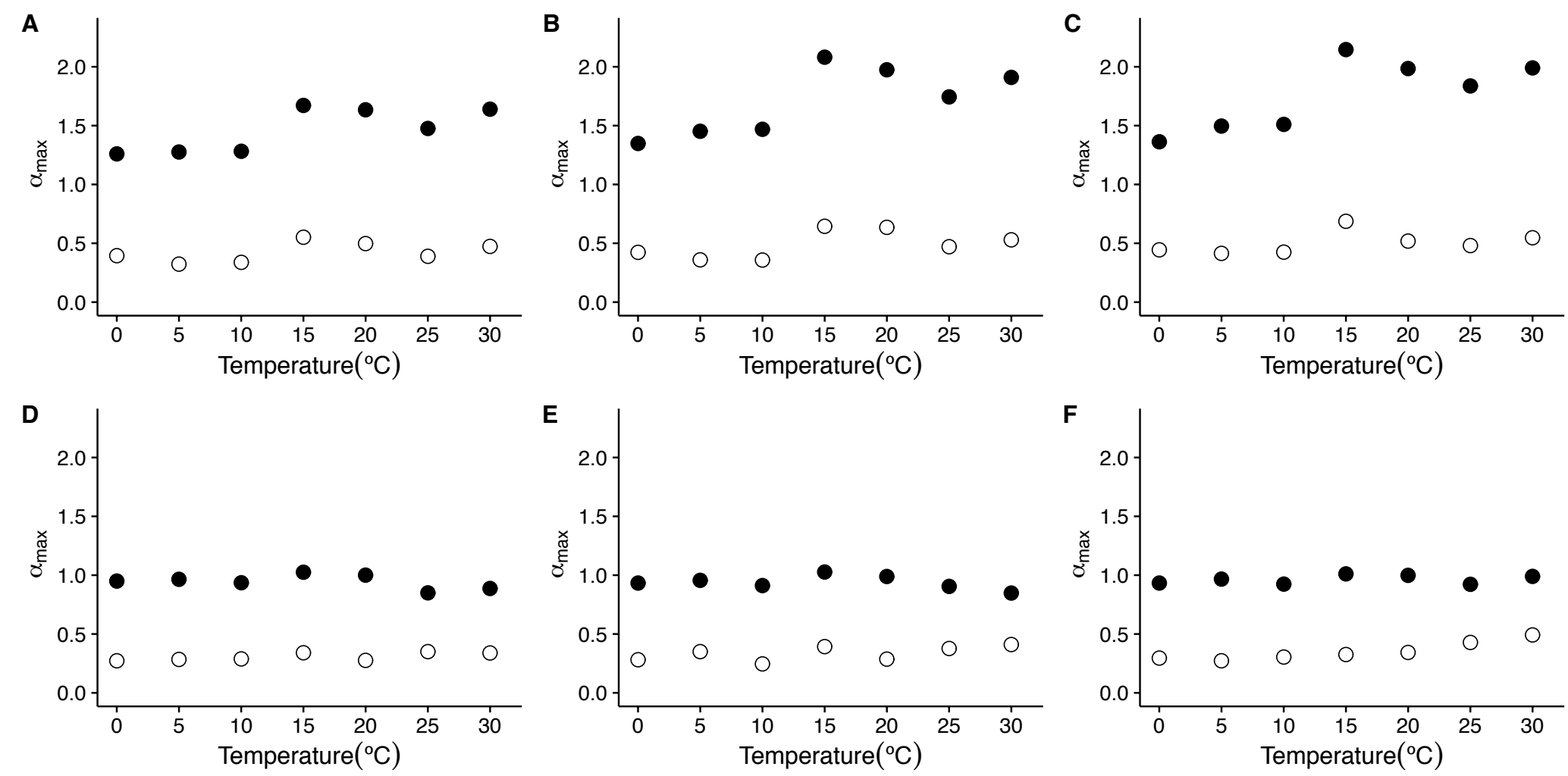